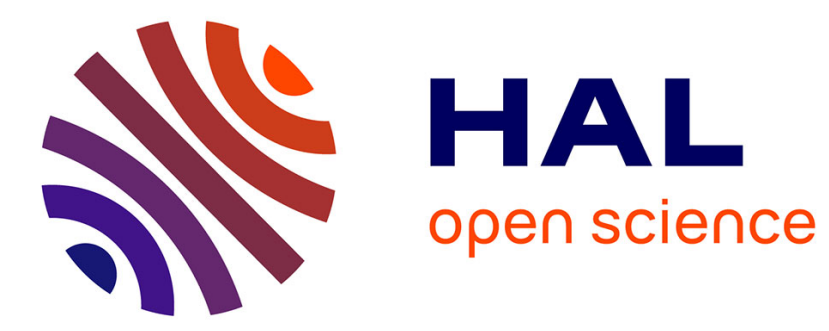

\title{
Rotation invariant, Riesz bases of directional wavelets Sylvain Durand
}

\section{To cite this version:}

Sylvain Durand. Rotation invariant, Riesz bases of directional wavelets. Applied and Computational Harmonic Analysis, 2017, 10.1016/j.acha.2017.04.001 . hal-01515774

\section{HAL Id: hal-01515774 https://hal.science/hal-01515774}

Submitted on 28 Apr 2017

HAL is a multi-disciplinary open access archive for the deposit and dissemination of scientific research documents, whether they are published or not. The documents may come from teaching and research institutions in France or abroad, or from public or private research centers.
L'archive ouverte pluridisciplinaire HAL, est destinée au dépôt et à la diffusion de documents scientifiques de niveau recherche, publiés ou non, émanant des établissements d'enseignement et de recherche français ou étrangers, des laboratoires publics ou privés. 


\title{
Rotation invariant, Riesz bases of directional wavelets.
}

\author{
Sylvain Durand \\ MAP5, CNRS UMR 8145, 45 rue des Saints Pères, 75270 Paris Cedex 06, France \\ e-mail: sylvain.durand@mi.parisdescartes.fr
}

April 28, 2017

\begin{abstract}
This article addresses the issue of designing bases for $L^{2}\left(\mathbb{R}^{2}\right)$ that are generated by translations, rotations and dilations of a single mother wavelet $\psi$. We show how this construction can be simplified by setting an odd number of directions and by choosing properly the phase of the Fourier transform of $\psi$. A large part of the article is devoted to the proof of theorems that give sufficient conditions for $\psi$ to generate a Riesz sequence and a Riesz basis for $L^{2}\left(\mathbb{R}^{2}\right)$. An example of Riesz sequence whose restriction to each scale is orthonormal is set. Theoretical results are confirmed by numerical experiments where a discrete directional wavelet transform is introduced.
\end{abstract}

\section{Introduction}

For more than two decades, the wavelet transform has been acknowledged as a very powerful tool in image processing. It is still widely used in many areas such as restoration and classification, but its efficiency is especially renowned for its applications in image compression through JPEG2000 [19], ICER [17] and CCSDS [6] standards.

As wavelets were originally designed for $1 \mathrm{D}$ signals, they have been extended to $2 \mathrm{D}$ images via tensor product. Since then, many transforms have been introduced as alternatives to the separable wavelet transform in order to represent more efficiently directional structures. The most famous examples include hexagonal wavelets [28, 8], steerable pyramids [29], directional wavelets [1], dual-tree complex wavelets [20, 27], ridgelets [4], curvelets [5,3], contourlets [9], shearlets [14], wavelets with composite dilations [15, 16] and related constructions such that Haar-type wavelets [18] or crystallographic wavelets $[2,21,22]$. All these transforms amount to express the image in a frame. But, except for orthonormal ridgelets [10] that are designed for images with straight edges, up to now only transforms which rely on a filterbank tree are known to be possibly non-redundant and can therefore be used in compression $[30,26,11,15,16$, 18, 12, 13, 2, 31, 22]. Filterbanks have well known digital advantages, but suffer the drawback that the decimation generates aliasing on the bases functions as it is shown in Figure 1-(a) that is extracted from [13], and it produces therefore artifacts on the compressed image. Thus, the design of directional bases that have all the required properties remains an open problem.

Notice that we did not mention adaptive multiscale transforms such as bandlets [23]. The second generation bandlets are non-redundant [24], but the separable wavelet basis used in their design could be replaced by any directional wavelet basis in order to get better approximations.

In this article, we focus on Antoine and Murenzi's directional wavelets [1, 25]. This sequence stands out from most of the other examples quoted above thanks to the simplicity of its construction. It is generated by a single wavelet $\psi$ in the Schwartz space $\mathcal{S}\left(\mathbb{R}^{2}\right)$ to which a composition of translations, 


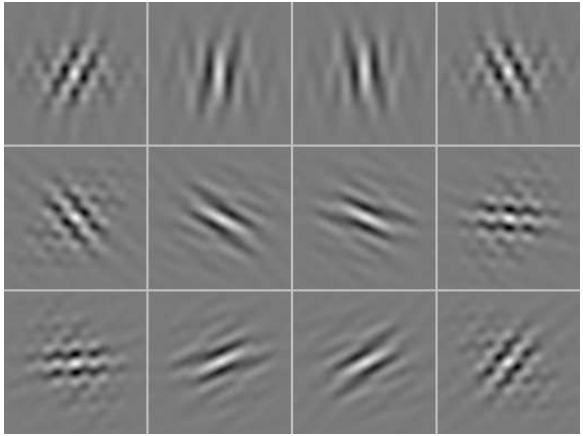

(a)

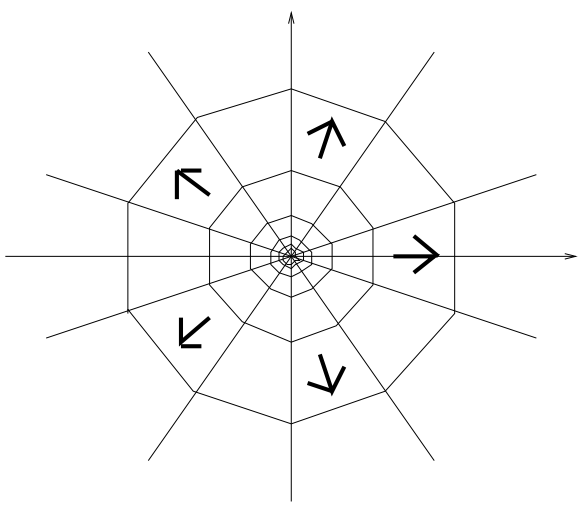

(b)

Figure 1: (a) Directional wavelets generated by a filterbank tree are aliased [13]. (b) The proposed directional wavelet basis has an odd number $N$ of directions, and is invariant under rotation of angle $\frac{2 \pi}{N}$.

dilations and rotations is applied. Denote by $R$ the rotation, by $\Gamma$ the regular lattice along which the wavelet is shifted, by $\Gamma^{*}$ its dual lattice and put $\psi_{j, k, \gamma}=2^{j} \psi\left(2^{j} R^{k} \cdot-\gamma\right)$. It is well known that if the function $\sum_{j \in \mathbb{Z}} \sum_{k \in\{0, \cdots, N-1\}}\left|\widehat{\psi}\left(2^{j} R^{k} \cdot\right)\right|^{2}$ is positive and if supp $\widehat{\psi}(\cdot) \cap \operatorname{supp} \widehat{\psi}(\cdot-\gamma)=\emptyset$, for all $\gamma \in \Gamma^{*}$, then $\left(\psi_{j, k, \gamma}\right)_{j \in \mathbb{Z}, k \in\{0, \cdots, N-1\}, \gamma \in \Gamma}$ is a frame for $L^{2}\left(\mathbb{R}^{2}\right)$.

It is generally believed that one cannot obtain bases for $L^{2}\left(\mathbb{R}^{2}\right)$ with this approach. This article aims to contradict this idea. More precisely, we show that, if the number of rotations $N$ is odd, the phase of the Fourier transform $\widehat{\psi}$ is well chosen and $\widehat{\psi}$ satisfies some specific inequalities, then the lattice $\Gamma$ can be changed into a larger one so that $\left(\psi_{j, k, \gamma}\right)_{(j, k, \gamma) \in \mathbb{Z} \times\{0, \cdots, N-1\} \times \Gamma}$ becomes surprisingly a Riesz sequence or possibly a Riesz basis for $L^{2}\left(\mathbb{R}^{2}\right)$.

\section{Notations and preliminaries}

\subsection{Notations}

All along this article, we will use the following notations.

- $\mu=(2 \pi, 0), \nu=\left(\pi, 3 \pi \tan \left(\frac{\pi}{2 N}\right)\right)$ and $\eta=\left(\pi, \pi \tan \left(\frac{\pi}{2 N}\right)\right)$ - see Figure 2.

- $\Gamma=\mathbb{Z}\left(1, \frac{-1}{3 \tan (\pi / 2 N)}\right)+\mathbb{Z}\left(0, \frac{2}{3 \tan (\pi / 2 N)}\right)$.

- The dual lattice of $\Gamma$ is therefore

$$
\Gamma^{*}=\left\{\gamma \in \mathbb{R}^{2}: \gamma \cdot \lambda \in 2 \pi \mathbb{Z}, \forall \lambda \in \Gamma\right\}=\mathbb{Z} \mu+\mathbb{Z} \nu
$$

- For all $\xi=\left(\xi_{1}, \xi_{2}\right) \in \mathbb{R}^{2}$, we denote by $\tilde{\xi}=\left(-\xi_{1}, \xi_{2}\right)$ its symmetrical point with respect to the ordinate axis.

- $R$ is the rotation of angle $\frac{(N-1) \pi}{N}$ about 0 . Observe that $R \eta=\tilde{\eta}$.

- $\mathcal{I}=\left\{\xi=\left(\xi_{1}, \xi_{2}\right) \in \mathbb{R}^{2}: \xi_{1} \in[-2 \pi,-\pi] \cup[\pi, 2 \pi]\right.$ and $\left.\left|\xi_{2}\right| \leq\left|\xi_{1}\right| \tan \left(\frac{\pi}{2 N}\right)\right\}$-see Figure 2. Notice that $(\mathcal{I}+\{\gamma\})_{\gamma \in \Gamma^{*}}$ and $\left(2^{j} R^{k} \mathcal{I}\right)_{j \in \mathbb{Z}, k \in\{0, \cdots, N-1\}}$ form tilings of $\mathbb{R}^{2}$.

- Denote by $\mathcal{L}$ the Lebesgue measure. For example, we have $\mathcal{L}(\mathcal{I})=6 \pi^{2} \tan \left(\frac{\pi}{2 N}\right)$. 


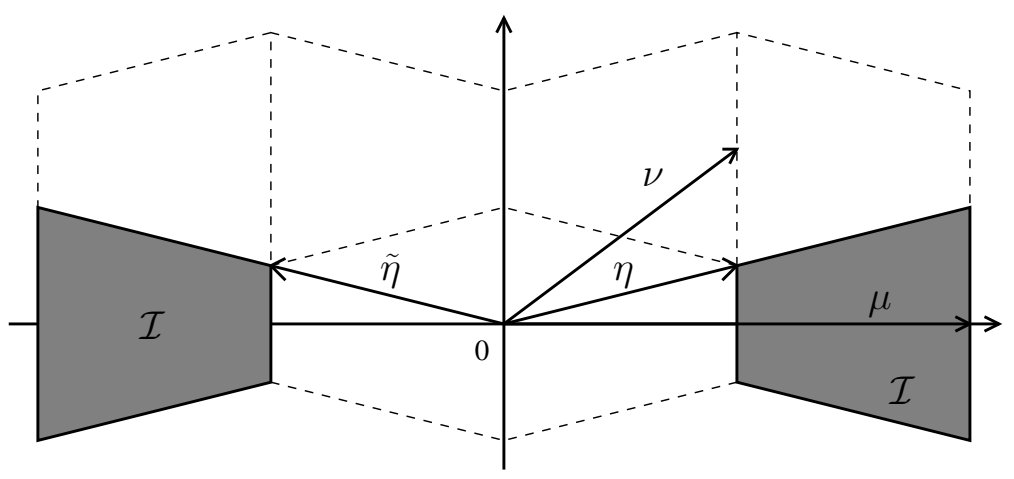

Figure 2: The set $\mathcal{I}$ represents the ideal frequency support of the wavelet $\psi$. The vectors $\mu$ and $\nu$ generates a lattice $\Gamma^{*}$ such that $(\mathcal{I}+\{\gamma\})_{\gamma \in \Gamma^{*}}$ forms a tiling of $\mathbb{R}^{2}$.

- The complex conjugate of any $z \in \mathbb{C}$ is denoted by $\bar{z}$.

- Denote by $\mathcal{S}\left(\mathbb{R}^{2}\right)$, the Schwartz space or, in other words, the space of functions all of whose derivatives are rapidly decreasing.

- The Fourier transform $\mathcal{F}$ is defined by

$$
\mathcal{F}(f)(\xi)=\hat{f}(\xi)=\int_{\mathbb{R}^{2}} f(x) e^{-i x \cdot \xi} d \xi, \quad \forall \xi \in \mathbb{R}^{2}, \forall f \in L^{1}\left(\mathbb{R}^{2}\right),
$$

and is extended to $L^{2}\left(\mathbb{R}^{2}\right)$ by continuity.

- $\psi_{j, k \gamma}=2^{j} \psi\left(2^{j} R^{k} \cdot-\gamma\right), \forall j \in \mathbb{Z}, \forall k \in \frac{\mathbb{Z}}{N \mathbb{Z}}, \forall \gamma \in \Gamma$.

- We denote by $\mathbb{I}_{A}$ the indicator function of any set $\mathcal{A} \in \mathbb{R}^{2}$, and by $\widehat{\psi}_{a}=\widehat{\psi} \mathbb{I}_{\left\{\xi_{1} \geq 0\right\}}$ and $\widehat{\psi}_{a a}=$ $\widehat{\psi} \mathbb{I}_{\left\{\xi_{1} \leq 0\right\}}$ the Fourier transforms of the generalized analytic and anti-analytic parts of $\psi \in \mathcal{S}\left(\mathbb{R}^{2}\right)$.

- For all $f, g \in L^{2}\left(\mathbb{R}^{2}\right)$, we denote by $\langle f, g\rangle=\int_{\mathbb{R}^{2}} f(x) \overline{g(x)} d x$, the scalar product on $L^{2}\left(\mathbb{R}^{2}\right)$.

\subsection{Shannon directional wavelets}

The Shannon directional wavelet basis is generated by a single wavelet $\psi=2 \pi(\mathcal{L}(\mathcal{I}))^{-\frac{1}{2}} \mathcal{F}^{-1}\left(\mathbb{I}_{\mathcal{I}}\right)$ - see above for notations. Let $R$ be any rotation about 0 such that $\left(2^{j} R^{k} \mathcal{I}\right)_{j \in \mathbb{Z}, k \in\{0, \cdots, N-1\}}$ forms a tiling of $\mathbb{R}^{2}$. It is natural to choose hitherto $\frac{\pi}{N}$ as the angle of rotation, but for reasons that will make clear later, we will use afterwards a rotation of angle $\frac{(N-1) \pi}{N}$.

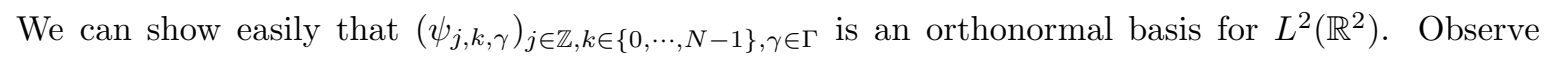
indeed that we have

$$
\mathcal{F}\left(\psi_{0,0, \gamma}\right)(\xi)=\mathcal{F}(\psi(\cdot-\gamma))(\xi)=\widehat{\psi}(\xi) e^{-i \gamma \cdot \xi}=\frac{2 \pi}{(\mathcal{L}(\mathcal{I}))^{\frac{1}{2}}} \mathbb{I}_{\mathcal{I}}(\xi) e^{-i \gamma \cdot \xi}, \quad \forall \gamma \in \Gamma, \xi \in \mathbb{R}^{2},
$$

where $\left(\xi \mapsto(\mathcal{L}(\mathcal{I}))^{-\frac{1}{2}} e^{-i \gamma \cdot \xi}\right)_{\gamma \in \Gamma}$ is an orthonormal basis for $L^{2}\left(\frac{\mathbb{R}^{2}}{\Gamma^{*}}\right)$ or equivalently for $L^{2}(\mathcal{I})$, since $(\mathcal{I}+\{\gamma\})_{\gamma \in \Gamma^{*}}$ is a tiling of $\mathbb{R}^{2}$. By Plancherel's theorem, $\left(\psi_{0,0, \gamma}\right)_{\gamma \in \Gamma}$ is therefore an orthonormal basis for $W_{0,0}^{s}=\left\{f \in L^{2}\left(\mathbb{R}^{2}\right): \operatorname{supp} \hat{f} \subset \mathcal{I}\right\}$. We show the same way that, for all $j \in \mathbb{Z}$ and $k \in\{0, \cdots, N-1\}$, $\left(\psi_{j, k, \gamma}\right)_{\gamma \in \Gamma}$ is an orthonormal basis for

$$
W_{j, k}^{s}=\left\{f \in L^{2}\left(\mathbb{R}^{2}\right): \operatorname{supp} \hat{f} \subset 2^{-j} R^{k} \mathcal{I}\right\}
$$


Then, we conclude by using the identity

$$
L^{2}\left(\mathbb{R}^{2}\right)=\bigoplus_{j \in \mathbb{Z}, k \in\{0, \cdots, N-1\}} W_{j, k}^{s},
$$

which comes from the fact that $\left(2^{j} R^{k} \mathcal{I}\right)_{j \in \mathbb{Z}, k \in\{0, \cdots, N-1\}}$ is a tiling of $\mathbb{R}^{2}$.

The reader is referred to [12] and the wavelets with composite dilations $[15,16]$ for more examples of Shannon wavelets. Shannon directional wavelets are of limited use since they have slow decay. It is therefore natural to raise the issue of designing an approximation of this sequence that keeps the structure of a basis, but which elements have fast decay.

\subsection{Riesz basis and frame}

As directional wavelets with fast decay and sharp frequency localization cannot form an orthonormal basis [13], we focus on a Riesz basis. Let $\mathcal{K}$ be a countable set. A sequence $\left(\psi_{k}\right)_{k \in \mathcal{K}}$ in $L^{2}\left(\mathbb{R}^{2}\right)$ is said to be a Riesz sequence if and only if there exists a constant $C \geq 1$ such that

$$
\frac{1}{C} \sum_{k \in \mathcal{K}}\left|a_{k}\right|^{2} \leq \int_{\mathbb{R}^{2}}\left|\sum_{k \in \mathcal{K}} a_{k} \psi_{k}(x)\right|^{2} d x \leq C \sum_{k \in \mathcal{K}}\left|a_{k}\right|^{2}, \quad \forall a \in \ell^{2}(\mathcal{K}) .
$$

It is said to be a Riesz basis for $L^{2}\left(\mathbb{R}^{2}\right)$ if and only if it is a Riesz sequence and a global sequence for $L^{2}\left(\mathbb{R}^{2}\right)$. In other words, we have also

$$
\operatorname{closure}\left(\operatorname{span}\left\{\psi_{k}: k \in \mathcal{K}\right\}\right)=L^{2}\left(\mathbb{R}^{2}\right) .
$$

The sequence $\left(\psi_{k}\right)_{k \in \mathcal{K}}$ is said to be a frame for $L^{2}\left(\mathbb{R}^{2}\right)$ if and only there exists a constant $C \geq 1$ such that

$$
\frac{1}{C} \sum_{k \in \mathcal{K}}\left|\left\langle f, \psi_{k}\right\rangle\right|^{2} \leq \int_{\mathbb{R}^{2}}|f(x)|^{2} d x \leq C \sum_{k \in \mathcal{K}}\left|\left\langle f, \psi_{k}\right\rangle\right|^{2}, \quad \forall f \in L^{2}\left(\mathbb{R}^{2}\right) .
$$

\section{Main results}

\subsection{Introductory example}

Before setting the main results, let us consider a specific example of directional wavelet. For $\tau>0$, let $H_{\tau}$ be a smooth approximation of the Heaviside function such that

$$
H_{\tau}^{2}(t)+H_{\tau}^{2}(-t)=1, \quad \forall t \in \mathbb{R}
$$

By smooth approximation, we mean that $H_{\tau}$ is a $\mathcal{C}^{\infty}$ and $[0,1]$-valued function such that $H_{\tau}(t)=0$, if $t \leq-\tau$, and 1, if $t \geq \tau$ - such a function is used in the design of Meyer wavelet. Thus, for a small $\tau>0$, the smooth function $V_{\tau}$ given by

$$
\begin{aligned}
V_{\tau}(\xi)= & H_{\tau}\left(\xi_{1} \sin \left(\frac{\pi}{2 N}\right)-\xi_{2} \cos \left(\frac{\pi}{2 N}\right)\right) H_{\tau}\left(\xi_{1} \sin \left(\frac{\pi}{2 N}\right)+\xi_{2} \cos \left(\frac{\pi}{2 N}\right)\right) \\
& +H_{\tau}\left(-\xi_{1} \sin \left(\frac{\pi}{2 N}\right)+\xi_{2} \cos \left(\frac{\pi}{2 N}\right)\right) H_{\tau}\left(-\xi_{1} \sin \left(\frac{\pi}{2 N}\right)-\xi_{2} \cos \left(\frac{\pi}{2 N}\right)\right)
\end{aligned}
$$

is drawn near to the indicator function of the set $\left\{\xi \in \mathbb{R}^{2}:\left|\xi_{2}\right| \leq\left|\xi_{1}\right| \tan \left(\frac{\pi}{2 N}\right)\right\}$, and for a small $\delta>0$, the function $H_{\delta}(|\cdot|-\pi) H_{\delta}(2 \pi-|\cdot|)$ is close to the indicator function of $[-2 \pi,-\pi] \cup[\pi, 2 \pi]$. Define therefore the wavelet $\psi$ as the inverse Fourier transform of $\widehat{\psi}$ given by

$$
\widehat{\psi}(\xi)=e^{\frac{i \xi_{1}}{2}} \frac{2 \pi}{\mathcal{L}(\mathcal{I})^{\frac{1}{2}}} H_{\delta}\left(\left|\xi_{1}\right|-\pi\right) H_{\delta}\left(2 \pi-\left|\xi_{1}\right|\right) V_{\tau}(\xi), \quad \forall \xi \in \mathbb{R}^{2} .
$$


When $\delta$ and $\tau$ tend to $0, \widehat{\psi}$ tends to $\left(\xi \mapsto e^{\frac{i \xi_{1}}{2}} 2 \pi \mathcal{L}(\mathcal{I})^{-\frac{1}{2}} \mathbb{I}_{\mathcal{I}}(\xi)\right)$, in the sense of the $L^{2}$ norm. The function $\psi$ tends therefore to the Shannon wavelet that has been translated by $\left(\frac{1}{2}, 0\right)$. We will make clearer the meaning of this translation-i.e. the term $e^{\frac{i \xi_{1}}{2}}$-later on.

On the other hand, this wavelet $\psi$ has been design in order to satisfy the following lemma

Lemma 1 Let $\psi$ be as in (2) and $R$ denote the rotation of angle $\frac{(N-1) \pi}{N}$. For all $\delta \in\left(0, \frac{\pi}{2}\right], \tau \in$ $\left(0,(\pi-\delta) \sin \left(\frac{\pi}{2 N}\right)\right]$ and $\xi \in \mathbb{R}^{2}$, we have

$$
\begin{array}{ll}
\text { (i) } & \sum_{\gamma \in \Gamma^{*}}|\widehat{\psi}(\xi-\gamma)|^{2}=\frac{(2 \pi)^{2}}{\mathcal{L}(\mathcal{I})}, \\
\text { (ii) } \quad & \left|\widehat{\psi_{a}}(R(\xi-3 \eta)) \widehat{\psi_{a a}}(\xi-3 \eta)\right|=\left|\widehat{\psi_{a a}}(R \xi) \widehat{\psi_{a}}(\xi)\right|,
\end{array}
$$

where $\widehat{\psi_{a}}=\mathbb{1}_{\left\{\xi_{1}>0\right\}} \widehat{\psi}$ and $\widehat{\psi_{a a}}=\mathbb{1}_{\left\{\xi_{1}<0\right\}} \widehat{\psi}$.

Identity $(i)$ allows to show that, for all $j \in \mathbb{Z}$ and $k \in\{0, \cdots, N-1\}$, the sequence $\left(\psi_{j, k, \gamma}\right)_{\gamma \in \Gamma}$ is orthonormal. Indeed, by Plancherel formula, we know that, for all $\alpha$ and $\beta \in \Gamma$,

$$
\left\langle\psi_{0,0, \alpha}, \psi_{0,0, \beta}\right\rangle=\langle\psi(\cdot-\alpha), \psi(\cdot-\beta)\rangle=\frac{1}{(2 \pi)^{2}}\langle\mathcal{F}(\psi(\cdot-\alpha)), \mathcal{F}(\psi(\cdot-\beta))\rangle,
$$

where $\mathcal{F}(\psi(\cdot-\alpha))(\xi)=\widehat{\psi}(\xi) e^{-i \alpha \cdot \xi}, \forall \xi \in \mathbb{R}^{2}$. The scalar product can therefore be expressed as

$$
\left\langle\psi_{0,0, \alpha}, \psi_{0,0, \beta}\right\rangle=\frac{1}{(2 \pi)^{2}} \int_{\mathbb{R}^{2}}|\widehat{\psi}(\xi)|^{2} e^{i(\beta-\alpha) \cdot \xi} d \xi=\frac{1}{(2 \pi)^{2}} \sum_{\gamma \in \Gamma^{*}} \int_{\mathcal{I}}|\widehat{\psi}(\xi-\gamma)|^{2} e^{i(\beta-\alpha) \cdot(\xi-\gamma)} d \xi
$$

because $(\mathcal{I}+\{\gamma\})_{\gamma \in \Gamma^{*}}$ is a tilling of $\mathbb{R}^{2}$. Since $e^{i(\beta-\alpha) \cdot \gamma}=1$, for all $\gamma \in \Gamma^{*}$, and $\widehat{\psi}$ satisfies $(i)$, we infer that

$$
\left\langle\psi_{0,0, \alpha}, \psi_{0,0, \beta}\right\rangle=\frac{1}{(2 \pi)^{2}} \int_{\frac{\mathbb{R}^{2}}{\Gamma^{*}}} \sum_{\gamma \in \Gamma^{*}}|\widehat{\psi}(\xi-\gamma)|^{2} e^{i(\beta-\alpha) \cdot \xi} d \xi=\frac{1}{\mathcal{L}(\mathcal{I})} \int_{\frac{\mathbb{R}^{2}}{\Gamma^{*}}} e^{i(\beta-\alpha) \cdot \xi} d \xi=\left\{\begin{array}{l}
1, \text { if } \alpha=\beta, \\
0, \text { otherwise. }
\end{array}\right.
$$

Thus, using changes of variables, we show that, for all $j \in \mathbb{Z}$ and $k \in\{0, \cdots, N-1\},\left(\psi_{j, k, \gamma}\right)_{\gamma \in \Gamma}$ is an orthonormal basis for the space $W_{j, k}:=\operatorname{closure}\left(\operatorname{span}\left(\psi_{j, k, \gamma}\right)_{\gamma \in \Gamma}\right)$.

¿From now on, suppose that the number of directions $N$ is odd. In such a case, we have $N \times \frac{(N-1) \pi}{N} \in$ $2 \pi \mathbb{Z}$ and $R^{N}$ is no more than the identity map. The rotation number $k$ can therefore be indexed now in $\frac{\mathbb{Z}}{N \mathbb{Z}}$ and we can show, by change of variables, that the product $\left\langle\psi_{0, k+1, \alpha}, \psi_{0, k, \beta}\right\rangle$ does not depends on $k$ in this set.

Hence, consider $\left\langle\psi_{0,1, \alpha}, \psi_{0,0, \beta}\right\rangle$. By the same arguments as above, we show that

$$
\left\langle\psi_{0,1, \alpha}, \psi_{0,0, \beta}\right\rangle=\frac{1}{(2 \pi)^{2}} \int_{\mathbb{R}^{2}} \widehat{\psi}(R \xi) \overline{\hat{\psi}(\xi)} e^{i(\beta \cdot \xi-\alpha \cdot R \xi)} d \xi
$$

Observe that the product of the two Fourier transforms can be developed as $\widehat{\psi}(R \xi) \overline{\widehat{\psi}(\xi)}=\widehat{\psi_{a a}}(R \xi) \overline{\widehat{\psi_{a}}(\xi)}+$ $\widehat{\psi_{a}}(R \xi) \overline{\widehat{\psi_{a a}}(\xi)}$. The scalar product becomes therefore

$$
\left\langle\psi_{0,1, \alpha}, \psi_{0,0, \beta}\right\rangle=\frac{1}{(2 \pi)^{2}} \int_{\mathbb{R}^{2}} \widehat{\psi_{a a}}(R \xi) \overline{\widehat{\psi_{a}}(\xi)} e^{i(\beta \cdot \xi-\alpha \cdot R \xi)} d \xi+\frac{1}{(2 \pi)^{2}} \int_{\mathbb{R}^{2}} \widehat{\psi_{a}}(R \xi) \overline{\widehat{\psi_{a a}}(\xi)} e^{i(\beta \cdot \xi-\alpha \cdot R \xi)} d \xi .
$$

Since $3 \eta=\left(3 \pi, 3 \pi \tan \frac{\pi}{2 N}\right)=\nu+\mu \in \Gamma^{*}$ and $R(3 \eta)=3 \tilde{\eta}=\left(-3 \pi, 3 \pi \tan \frac{\pi}{2 N}\right)=\nu-2 \mu \in \Gamma^{*}$, we have $e^{i(\beta \cdot(3 \eta)-\alpha \cdot R(3 \eta))}=1$, and if we change the variable $\xi$ into $\xi-3 \eta$, in the second integral, we obtain

$$
\left\langle\psi_{0,1, \alpha}, \psi_{0,0, \beta}\right\rangle=\frac{1}{(2 \pi)^{2}} \int_{\mathbb{R}^{2}}\left(\widehat{\psi_{a a}}(R \xi) \overline{\widehat{\psi_{a}}(\xi)}+\widehat{\psi_{a}}(R(\xi-3 \eta)) \overline{\left.\widehat{\psi_{a a}}(\xi-3 \eta)\right)} e^{i(\beta \cdot \xi-\alpha \cdot R \xi)} d \xi .\right.
$$


Notice next that $(R \eta)_{1}-\eta_{1}=\tilde{\eta}_{1}-\eta_{1}=-\pi$. Hence, $e^{i \frac{3(R \eta)_{1}}{2}} e^{-i \frac{3 \eta_{1}}{2}}=e^{-i \frac{3 \pi}{2}}=-1$ and

$$
\begin{aligned}
\widehat{\psi_{a}}(R(\xi-3 \eta)) \widehat{\widehat{\psi_{a a}}(\xi-3 \eta)} & =e^{i \frac{(R \xi)_{1}-3(R \eta)_{1}}{2}} e^{-i \frac{\xi_{1}-3 \eta_{1}}{2}}\left|\widehat{\psi_{a}}(R(\xi-3 \eta)) \widehat{\widehat{\psi_{a a}}(\xi-3 \eta)}\right| \\
& =-e^{i \frac{(R \xi)_{1}}{2}} e^{-i \frac{\xi_{1}}{2}}\left|\widehat{\psi_{a}}(R(\xi-3 \eta)) \widehat{\widehat{\psi_{a a}}(\xi-3 \eta)}\right| \\
& =-\widehat{\psi_{a a}}(R \xi) \widehat{\widehat{\psi_{a}}(\xi)}
\end{aligned}
$$

thanks to $(i i)$. We deduce that $\left\langle\psi_{0,1, \alpha}, \psi_{0,0, \beta}\right\rangle=0, \forall \alpha, \beta \in \Gamma$. By symmetry, we have also $\left\langle\psi_{0,-1, \alpha}, \psi_{0,0, \beta}\right\rangle=$ 0 . As $\widehat{\psi}$ and $\widehat{\psi}\left(R^{k} \cdot\right)$ have disjoint supports whenever $\{-1,0,1\} \cap k=\emptyset$, we know therefore that $\left\langle\psi_{0, k, \alpha}, \psi_{0,0, \beta}\right\rangle=0, \forall k \in \frac{\mathbb{Z}^{2}}{N \mathbb{Z}^{2}} \backslash N \mathbb{Z}^{2}$. By changes of variables, we obtain also $\left\langle\psi_{j, k, \alpha}, \psi_{j, l, \beta}\right\rangle=0$, $\forall j \in \mathbb{Z}$, whenever $k \neq l$ and we have proven the following proposition.

Proposition 1 If $N$ is an odd number, $R$ is the rotation of angle $\frac{(N-1) \pi}{N}$ and $\psi$ is as in (2) with $\delta \in\left(0, \frac{\pi}{2}\right]$ and $\tau \in\left(0,(\pi-\delta) \sin \left(\frac{\pi}{2 N}\right)\right]$, then, for all $j \in \mathbb{Z},\left(\psi_{j, k, \gamma}\right)_{k \in \frac{\mathbb{R}^{2}}{\Gamma^{*}}, \gamma \in \Gamma}$ is an orthonormal basis for the space $W_{j}:=\bigoplus_{k \in \frac{\mathbb{Z}}{N \mathbb{Z}}} W_{j, k}$.

The spaces $W_{j}$ and $W_{j+1}$ cannot be orthogonal or, in other words, the whole sequence $\left(\psi_{j, k, \gamma}\right)_{j \in \mathbb{Z}, k \in \frac{\mathbb{R}^{2}}{\Gamma^{*}}, \gamma \in \Gamma^{*}}$ cannot be orthogonal-see [13]. Using similar arguments as above, we can easily show that, for all $k \in \frac{\mathbb{R}^{2}}{\Gamma^{*}}$, the angle between the spaces $W_{j, k}$ and $W_{j+1, k}$ is sufficiently large so that $\left(\psi_{j, k, \gamma}\right)_{j \in \mathbb{Z}, \gamma \in \Gamma}$ is a Riesz sequence. One of the questions that motivate this article is to know whether the whole sequence $\left(\psi_{j, k, \gamma}\right)_{j \in \mathbb{Z}, k \in \frac{\mathbb{R}^{2}}{\Gamma^{*}}, \gamma \in \Gamma^{*}}$ can be a Riesz sequence or a Riesz basis for $L^{2}\left(\mathbb{R}^{2}\right)$. We will study this sequence for a more general choice of wavelet $\psi$.

\subsection{Sufficient conditions for a Riesz sequence}

As a first step, we give sufficient conditions on a wavelet $\psi \in \mathcal{S}\left(\mathbb{R}^{2}\right)$ for its dilated, rotated and translated versions to generate a Riesz sequence. Conditions for a Riesz basis for $L^{2}\left(\mathbb{R}^{2}\right)$ are considered in the next section.

Let us make the following hypotheses.

$\mathbf{H 1}$ The number of directions $N$ is odd and $R$ is a rotation of angle $\frac{(N-1) \pi}{N}$ about 0 .

H2 We have $\widehat{\psi}(\xi)=e^{\frac{i \xi_{1}}{2}}|\widehat{\psi}(\xi)|, \forall \xi \in \mathbb{R}^{2}$.

H3 We have supp $\widehat{\psi} \cap \operatorname{supp} \widehat{\psi}\left(2^{j} R^{k} \cdot\right)=\emptyset$, whenever $|j|>1$ or $k \notin\{-1,0,1\}+N \mathbb{Z}$, and we have $|\widehat{\psi}(\xi)|=|\widehat{\psi}(-\xi)|=|\widehat{\psi}(\tilde{\xi})|, \forall \xi \in \mathbb{R}^{2}$.

H4 We have

$$
\Psi_{1}(\xi)+\Psi_{2}(\xi)+\Psi_{3}(\xi)<\Psi_{4}(\xi)+\sum_{\gamma \in \Gamma^{*}}|\widehat{\psi}(\xi+\gamma)|^{2}, \quad \forall \xi \in \mathbb{R}^{2}
$$

where

$$
\begin{aligned}
\Psi_{1}(\xi)= & \sum_{\gamma \in \Gamma^{*}}\left\|\widehat{\psi}_{a}(\xi+\gamma) \widehat{\psi}_{a}(2(\xi+\gamma))|-| \widehat{\psi}_{a a}(\xi+\gamma-\mu) \widehat{\psi}_{a a}(2(\xi+\gamma-\mu))\right\|, \\
& +\sum_{\gamma \in \Gamma^{*}}|| \widehat{\psi}_{a}(\xi+\gamma) \widehat{\psi}_{a}\left(2^{-1}(\xi+\gamma)\right)|-| \widehat{\psi}_{a a}(\xi+\gamma-2 \mu) \widehat{\psi}_{a a}\left(2^{-1}(\xi+\gamma-2 \mu)\right) \|, \\
\Psi_{2}(\xi)= & \sum_{\gamma \in \Gamma^{*}}\left\|\widehat{\psi}_{a}(\xi+\gamma) \widehat{\psi}_{a a}(R(\xi+\gamma))|-| \widehat{\psi}_{a a}(\xi+\gamma+3 \tilde{\eta}) \widehat{\psi}_{a}(R(\xi+\gamma+3 \tilde{\eta}))\right\|
\end{aligned}
$$



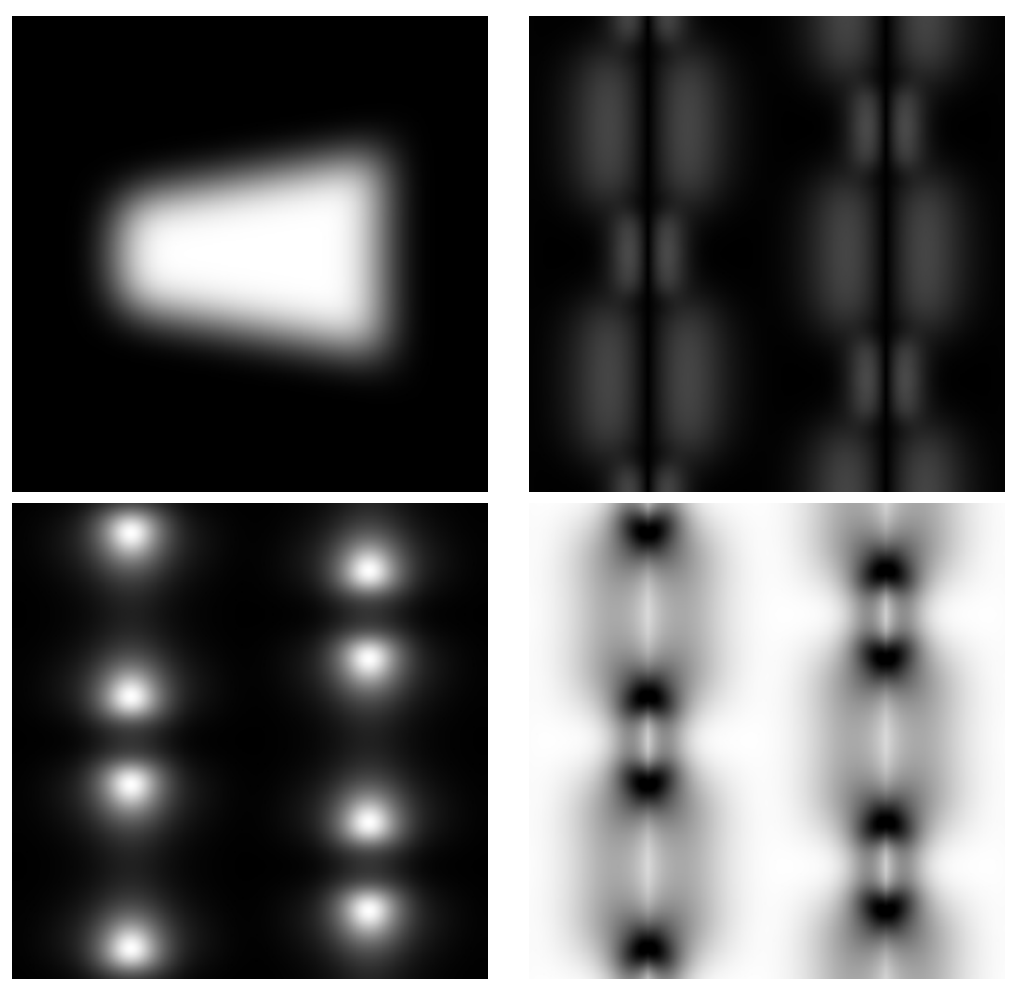

Figure 3: Functions $\left|\widehat{\psi}_{a}\right|, \Psi_{1}, \Psi_{3}$ and $\Psi_{4}+\sum_{\gamma \in \Gamma^{*}}|\widehat{\psi}(\cdot+\gamma)|^{2}-\Psi_{1}-\Psi_{2}-\Psi_{3}$ for $\psi$ as in $(2)$ and $N=9$. Only restrictions to the domain $[\pi / 2,5 \pi / 2] \times[-\pi, \pi]$ are shown.

$$
\begin{gathered}
+\sum_{\gamma \in \Gamma^{*}}|| \widehat{\psi}_{a}(\xi+\gamma) \widehat{\psi}_{a a}\left(R^{-1}(\xi+\gamma)\right)|-| \widehat{\psi}_{a a}(\xi+\gamma-3 \eta) \widehat{\psi}_{a}\left(R^{-1}(\xi+\gamma-3 \eta)\right) \|, \\
\Psi_{3}(\xi)=\sum_{j= \pm 1} \sum_{k= \pm 1} \sum_{\gamma \in \Gamma^{*}}\left|\widehat{\psi}(\xi+\gamma) \widehat{\psi}\left(2^{j} R^{k}(\xi+\gamma)\right)\right|, \\
\Psi_{4}(\xi)=2\left(1-\cos \frac{\pi}{2 N}\right) \\
\times \sum_{\epsilon= \pm 1} \sum_{\gamma \in \Gamma^{*}}\left(\min _{\left\{\omega \in \mathbb{R}^{2}:|\omega-\epsilon \eta|=|\xi-\epsilon \eta-\gamma|\right\}}|\widehat{\psi}(\omega) \widehat{\psi}(2 R \omega)|+\min _{\left\{\omega \in \mathbb{R}^{2}:|\omega-2 \epsilon \eta|=|\xi-2 \epsilon \eta-\gamma|\right\}}\left|\widehat{\psi}\left(2^{-1} \omega\right) \widehat{\psi}(R \omega)\right|\right) .
\end{gathered}
$$

Then we have the following theorem, the proof of which constitutes a large part of the remaining of this article.

Theorem 1 Let $\psi \in \mathcal{S}\left(\mathbb{R}^{2}\right)$. If conditions H1-4 are satisfied, then $\left(\psi_{j, k, \gamma}\right)_{(j, k, \gamma) \in \mathbb{Z} \times \frac{Z}{N Z} \times \Gamma}$ is a Riesz sequence.

Before going further, let us make some comments on the hypotheses itemized above. First, H1 and $\mathbf{H 2}$ are closely tied in the sense that one cannot enforce the dephasing of $\mathbf{H 2}$ if $N$ is even. Indeed, in order to maintain the invariance by rotation, despite the asymmetry caused by $\mathbf{H 2}$, two "neighboring" wavelets at the same scale must have nearly opposite directions as it is shown in Figure 1-(b). This is not possible if $N$ is even. As for the Meyer wavelet, there are several possible choice for the modulation. We chose $e^{i \frac{\xi_{1}}{2}}$ as it is often the case for the Meyer wavelet. Regarding the rotation $R$, any angle in 


\begin{tabular}{|l|c|c|c|c|}
\hline$N$ (number of direction) & 5 & 7 & 9 & 11 \\
\hline $\min _{\mathbb{R}^{2}} \frac{\mathcal{L}(\mathcal{I})}{(2 \pi)^{2}}\left(\sum_{\gamma \in \Gamma^{*}}|\widehat{\psi}(\cdot+\gamma)|^{2}-\Psi_{1}-\Psi_{2}-\Psi_{3}\right)$ & -0.001 & -0.007 & -0.016 & -0.024 \\
$\max _{\mathbb{R}^{2}} \frac{\mathcal{L}(\mathcal{I})}{(2 \pi)^{2}} \Psi_{4}=2\left(1-\cos \frac{\pi}{2 N}\right)$ & 0.098 & 0.050 & 0.030 & 0.020 \\
$\min _{\mathbb{R}^{2}} \frac{\mathcal{L}(\mathcal{I})}{(2 \pi)^{2}}\left(\sum_{\gamma \in \Gamma^{*}}|\widehat{\psi}(\cdot+\gamma)|^{2}-\Psi_{1}-\Psi_{2}-\Psi_{3}+\Psi_{4}\right)$ & $\mathbf{0 . 0 9 7}$ & $\mathbf{0 . 0 4 2}$ & $\mathbf{0 . 0 1 3}$ & -0.005 \\
\hline
\end{tabular}

Table 1: If $\psi$ is as in (2) with $\delta=\tau=\left(\pi \sin \frac{\pi}{2 N}\right) /\left(1+\sin \frac{\pi}{2 N}\right)$, the hypothesis $\mathbf{H} 4$ is satisfied for $N \leq 9$.

$\frac{2 \pi}{N}(\mathbb{Z} \backslash(N \mathbb{Z}))$ would be appropriate, but angles $\frac{(N-1) \pi}{N}$ or $\frac{(N+1) \pi}{N}$ let us express hypothesis $\mathbf{H} 4$ more easily.

Condition $\mathbf{H 3}$ is a technical, clearly not necessary, hypothesis. Its aim is to simplify $\mathbf{H} \mathbf{4}$ and the proof of the theorem. The only reason for assuming that $|\widehat{\psi}|$ is symmetric is to simplify the term $\Psi_{4}(\xi)$. This condition is however quite natural.

Hypothesis $\mathbf{H} 4$ is definitely the most difficult to deal with. Roughly speaking, $\Psi_{1}$ and $\Psi_{2}$ measure the angles between the spaces $W_{0,0}$ and $W_{1,0}$, and between $W_{0,0}$ and $W_{0,1}$, respectively. For instance, if $\Psi_{2}=0$, then the spaces $W_{0, k}$ are two-by-two orthogonal as it is the case in the example considered in Section 3.1. The functions $\Psi_{3}$ and $\Psi_{4}$ handle the direction of $W_{0,0}$ with regard to $W_{1,1}$ and $W_{1,-1}$. The proof of the theorem would be much simpler if we could discard $\Psi_{4}$ in the inequality (3). But in such a case, we cannot ensure that there exists any wavelet $\psi \in \mathcal{S}\left(\mathbb{R}^{2}\right)$ that satisfy all the hypotheses. For example, if $\psi$ is as above, then $|\widehat{\psi}( \pm \eta)|=|\widehat{\psi}( \pm 2 \eta)|=|\widehat{\psi}( \pm \tilde{\eta})|=|\widehat{\psi}( \pm 2 \tilde{\eta})|=\frac{1}{2} \mathcal{L}(\mathcal{I})^{-\frac{1}{2}}$. We infer that $\Psi_{1}(\eta)=0=\Psi_{2}(\eta)$, but $\Psi_{3}(\eta)=(2 \pi)^{2} / \mathcal{L}(\mathcal{I})=\sum_{\gamma \in \Gamma^{*}}|\widehat{\psi}(\eta+\gamma)|^{2}$.

The wavelet defined in (2) satisfies clearly $\mathbf{H 2}$ and $\mathbf{H 3}$ when $\delta$ and $\tau$ are as in Lemma 1. We do not have an analytic proof that it satisfies $\mathbf{H} 4$. We can however check digitally whether the inequality is satisfied but for quantization and sampling approximations. We know that $\sum_{\gamma \in \Gamma^{*}}|\widehat{\psi}(\cdot-\gamma)|^{2}=(2 \pi)^{2} / \mathcal{L}(\mathcal{I})$ and $\Psi_{2}=0$. Figure 3 shows $|\widehat{\psi}|, \Psi_{1}, \Psi_{3}$ and $\Psi_{4}+\sum_{\gamma \in \Gamma^{*}}|\widehat{\psi}(\cdot+\gamma)|^{2}-\Psi_{1}-\Psi_{2}-\Psi_{3}=\Psi_{4}+(2 \pi)^{2} / \mathcal{L}(\mathcal{I})-\Psi_{1}-\Psi_{3}$. The function $\Psi_{4}$ is not shown because it is close to $2\left(1-\cos \frac{\pi}{2 N}\right) \Psi_{3}$. The function $1-\mathcal{L}(\mathcal{I})\left(\Psi_{1}+\Psi_{3}\right) /(2 \pi)^{2}$ is positive outside a small neighborhood of $\{\eta,-\eta\}+\Gamma^{*}$ but its minimum value on $\mathbb{R}^{2}$ is negative. This value is given in Table 1 , for $N=5,7,9$ and 11 , as well as $\max _{\mathbb{R}^{2}} \mathcal{L}(\mathcal{I}) \Psi_{4} /(2 \pi)^{2}$ and $\min _{\mathbb{R}^{2}} 1-\mathcal{L}(\mathcal{I})\left(\Psi_{1}+\right.$ $\left.\Psi_{3}-\Psi_{4}\right) /(2 \pi)^{2}$. We can see that the hypothesis $\mathbf{H} 4$ is satisfied for $N=5,7$ and 9 , but it fails to be satisfied for $N=11$. In this example, we set $\delta=\tau=\left(\pi \sin \frac{\pi}{2 N}\right) /\left(1+\sin \frac{\pi}{2 N}\right)$ and $H_{\tau}(t)=\sin \left(\frac{\pi}{2} P\left(\frac{t-\tau}{2 \tau}\right)\right)$, $\forall t \in[-\tau, \tau]$, where $P(t)=35 t^{4}-84 t^{5}+70 t^{6}-20 t^{7}$. The obtained function $\widehat{\psi}$ is only $\mathcal{C}^{3}$ but it can be uniformly approximated by a $\mathcal{C}^{\infty}$ function that satisfies $\mathbf{H} 4$ also.

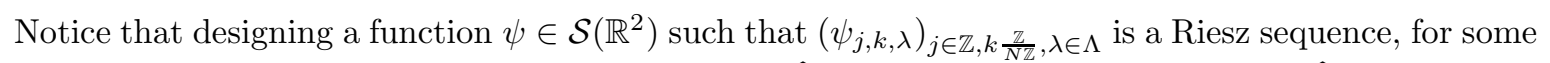
lattice $\Lambda$, is not a difficult issue. For instance, if supp $\widehat{\psi} \subset \mathcal{I}$ and $\Lambda$ is such that $\sum_{\lambda \in \Lambda^{*}}|\widehat{\psi}(\xi-\lambda)|^{2}>0$, then $\left(\psi_{j, k, \lambda}\right)_{j \in \mathbb{Z}, k \frac{\mathbb{Z}}{N Z}, \lambda \in \Lambda}$ is a Riesz sequence. Indeed, the second assumption ensures that $\left(\psi_{j, k, \lambda}\right)_{\lambda \in \Lambda}$ is a Riesz basis for closure $\left(\operatorname{span}\left(\psi_{j, k, \gamma}\right)_{\gamma \in \Lambda}\right)$, for all $j \in \mathbb{Z}$ and $k \in \frac{\mathbb{Z}}{N \mathbb{Z}}$, and by the first assumption, these spaces are two-by-two orthogonal, since they correspond to disjoint Fourier supports. However, in such a case, it is clear that the sequence $\left(\psi_{j, k, \lambda}\right)_{j \in \mathbb{Z}, k \frac{Z}{\mathrm{NZ}}, \lambda \in \Lambda}$ cannot be global for $L^{2}\left(\mathbb{R}^{2}\right)$. A notable point of Theorem 1 is that $\psi$ is translated along the critical lattice $\Gamma$ of the Shannon wavelet basis. Hence, the condition $\sum_{\gamma \in \Gamma^{*}}|\widehat{\psi}(\xi-\gamma)|^{2}>0, \forall \xi \in \mathbb{R}^{2}$, does not allow to have supp $\widehat{\psi} \subset \mathcal{I}$. 


\subsection{Sufficient conditions for a global sequence and a Riesz basis for $L^{2}\left(\mathbb{R}^{2}\right)$}

So far, we gave conditions on $\psi$ so that $\left(\psi_{j, k, \gamma}\right)_{j \in \mathbb{Z}, k \in \frac{Z}{N Z}, \gamma \in \Gamma}$ forms a Riesz basis for the closure of

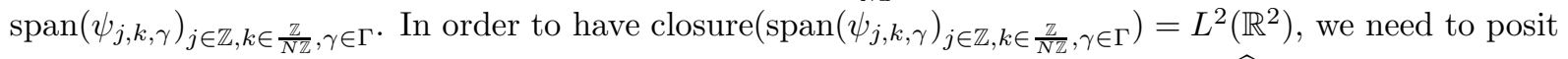
additional hypotheses. First, we need to narrow the conditions on the support of $\widehat{\psi}$ by assuming that it satisfies, besides H3, the following property which means that the supports of $\widehat{\psi}$ and $\widehat{\psi}(\cdot-\gamma)$ are disjoint, for all $\gamma \in \Gamma^{*}$ such that the distance between $\mathcal{I}$ and $\mathcal{I}+\{\gamma\}$ is positive.

H5 We have $\widehat{\psi}(\cdot) \widehat{\psi}(\cdot-\gamma)=0$, for all $\gamma \in \Gamma^{*} \backslash\{0, \mu,-\mu, 2 \mu,-2 \mu, 3 \eta,-3 \eta, 3 \tilde{\eta},-3 \tilde{\eta}, \nu,-\nu, \tilde{\nu},-\tilde{\nu}\}$.

As H3, condition $\mathbf{H 5}$ is not necessary, but it allows to simplify $\mathbf{H 6}$, below, that is the counterpart of H4 for a global sequence.

H6 We have

$$
\Phi_{1}(\xi)+\Phi_{2}(\xi)+\Phi_{3}(\xi)<\Phi_{4}(\xi)+\sum_{j \in \mathbb{Z}} \sum_{k \in \frac{Z}{N Z}}\left|\widehat{\psi}\left(2^{j} R^{k} \xi\right)\right|^{2}, \quad \text { for almost every } \xi \in \mathbb{R}^{2}
$$

where

$$
\begin{aligned}
& \Phi_{1}(\xi)=\sum_{\gamma= \pm \mu} \sum_{j \in \mathbb{Z}} \sum_{k \in \frac{\mathbb{Z}}{N \mathbb{Z}}}\left\|\widehat{\psi}\left(2^{j} R^{k} \xi\right) \widehat{\psi}\left(2^{j} R^{k} \xi-\gamma\right)|-| \widehat{\psi}\left(2^{j+1} R^{k} \xi\right) \widehat{\psi}\left(2^{j+1} R^{k} \xi-2 \gamma\right)\right\|, \\
& \Phi_{2}(\xi)=\sum_{\gamma= \pm 3 \eta} \sum_{j \in \mathbb{Z}} \sum_{k \in \frac{\mathbb{Z}}{N \mathbb{Z}}}\left\|\widehat{\psi}\left(2^{j} R^{k} \xi\right) \widehat{\psi}\left(2^{j} R^{k} \xi-\gamma\right)|-| \widehat{\psi}\left(2^{j} R^{k+1} \xi\right) \widehat{\psi}\left(2^{j} R^{k+1} \xi-R \gamma\right)\right\| \text {, } \\
& \Phi_{3}(\xi)=\sum_{\gamma \in\{-\nu, \nu,-\tilde{\nu}, \tilde{\nu}\}} \sum_{j \in \mathbb{Z}} \sum_{k \in \mathbb{Z}}\left|\widehat{\psi}\left(2^{j} R^{k} \xi\right) \widehat{\psi}\left(2^{j} R^{k} \xi-\gamma\right)\right|, \\
& \Phi_{4}(\xi)=4\left(1-\cos \frac{\pi}{2 N}\right) \sum_{\gamma \in\{2 \eta,-\tilde{\eta}\}} \sum_{j \in \mathbb{Z}} \sum_{k \in \frac{Z}{N Z}}\left\{\omega \in \mathbb{R}^{2}:|\omega|=\left|2^{-j} \xi-R^{k} \gamma\right|\right\}|\widehat{\psi}(2 \eta+\omega) \widehat{\psi}(-\tilde{\eta}+\omega)| .
\end{aligned}
$$

We can now state the announced theorem and corollary.

Theorem 2 Let $\psi \in \mathcal{S}\left(\mathbb{R}^{2}\right)$. If conditions H1-2 and H5-6 are satisfied, then $\left(\psi_{j, k, \gamma}\right)_{(j, k, \gamma) \in \mathbb{Z} \times \frac{\mathbb{Z}}{N \mathbb{Z}} \times \Gamma}$ is a global sequence for $L^{2}\left(\mathbb{R}^{2}\right)$. If, moreover, $\mathbf{H 6}$ is satisfied for all $\xi \in \mathbb{R}^{2}$, then $\left(\psi_{j, k, \gamma}\right)_{(j, k, \gamma) \in \mathbb{Z} \times \frac{\mathbb{Z}}{N \mathbb{Z}} \times \Gamma}$ is a frame for $L^{2}\left(\mathbb{R}^{2}\right)$.

Joining both theorems together, we obtain the following.

Corollary 1 Let $\psi \in \mathcal{S}\left(\mathbb{R}^{2}\right)$. If conditions H1-6 are satisfied, then $\left(\psi_{j, k, \gamma}\right)_{(j, k, \gamma) \in \mathbb{Z} \times \frac{\mathbb{Z}}{N Z} \times \Gamma}$ is a Riesz basis for $L^{2}\left(\mathbb{R}^{2}\right)$.

If $\delta$ and $\tau$ are sufficiently small, then the wavelet defined in (2) satisfies $\mathbf{H 5 .}$. With regard to $\mathbf{H 6}$, one can check that, for all $\gamma \in\left\{ \pm 2^{j} R^{k} \eta: j \in \mathbb{Z}, k \in \frac{\mathbb{Z}}{N \mathbb{Z}}\right\}$, we have $\sum_{\gamma \in \Gamma^{*}}\left|\widehat{\psi}\left(2^{j} R^{k} \gamma\right)\right|^{2}=(2 \pi)^{2} / \mathcal{L}(\mathcal{I})=$ $\Phi_{3}(\gamma)$, while $\Phi_{1}(\gamma)=\Phi_{2}(\gamma)=0$ and $\Phi_{4}(\gamma)>0$. We infer that $\Phi_{4}(\gamma)+\sum_{\gamma \in \Gamma^{*}}\left|\widehat{\psi}\left(2^{j} R^{k} \gamma\right)\right|^{2}-\Phi_{1}(\gamma)-$ $\Phi_{2}(\gamma)-\Phi_{3}(\gamma)>0$. The latter is also clearly positive whenever $\gamma$ is far from $\left\{ \pm 2^{j} R^{k} \eta: j \in \mathbb{Z}, k \in \frac{\mathbb{Z}}{N \mathbb{Z}}\right\}$. However, its minimum value on $\mathbb{R}^{2}$ is slightly below 0 . Hence the hypothesis $\mathbf{H 6}$ is not satisfied for this example. Figure 4 shows $\sum_{k \in \frac{Z}{N Z}}\left|\widehat{\psi}\left(2^{j} R^{k} \cdot\right)\right|^{2}, \Phi_{2}, \Phi_{3}$ and $\Phi_{4}+\sum_{\gamma \in \Gamma^{*}}\left|\widehat{\psi}\left(2^{j} R^{k} \cdot\right)\right|^{2}-\Phi_{1}-\Phi_{2}-\Phi_{3}$, for $N=9$. The function $\Phi_{1}$ is zero and $\Phi_{4}$ is closed to $2\left(1-\cos \frac{\pi}{2 N}\right) \Phi_{3}$. 

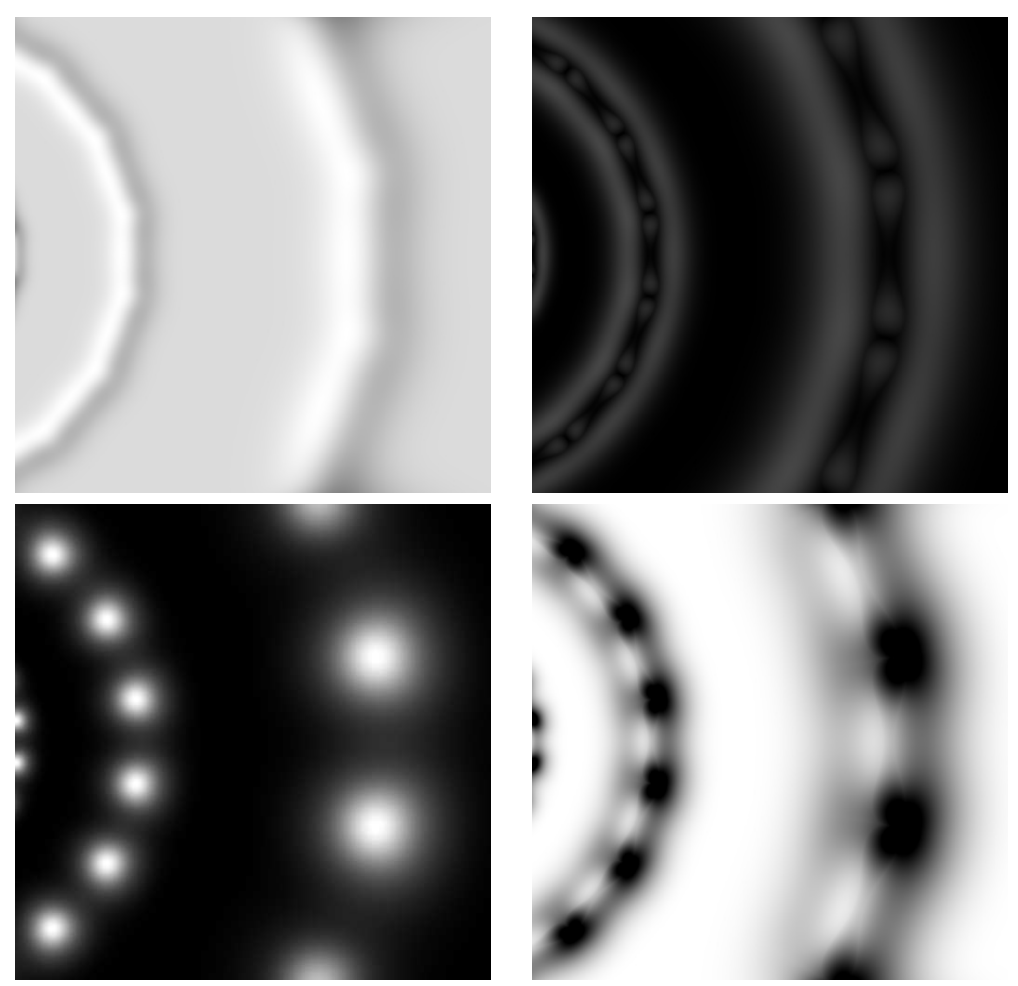

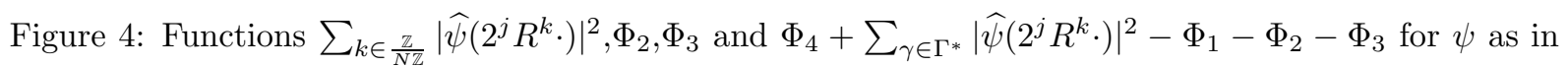
(2) and $N=9$. Only restrictions to the domain $[\pi / 2,5 \pi / 2] \times[-\pi, \pi]$ are shown.

We did not find a simple example of wavelet that satisfies all the six hypotheses. We cannot however conclude from this that the wavelet defined in (2), or any other wavelet, does not generate a basis for $L^{2}\left(\mathbb{R}^{2}\right)$. We only gave indeed sufficient condition for a global sequence. Although it is rather long, the proof of the theorems relies on coarse bounds and we can therefore conjecture that, when all the other hypotheses are satisfied, conditions $\mathbf{H 4}$ and $\mathbf{H 6}$ are not necessary. This proof of both theorems is detailed in the next section.

\section{Proofs}

\subsection{Proof of Theorem 1}

Since the demonstration is carried out in the Fourier domain, we have to give a characterization of the Riesz sequence in terms of the Fourier transform $\widehat{\psi}$. The following lemma is proved in Section 4.3.

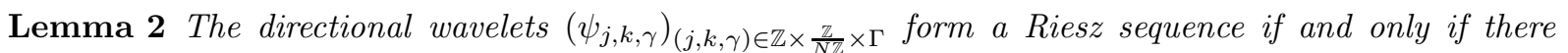
exists $C \geq 1$ such that, for all $m \in L^{2}\left(\mathbb{Z} \times \frac{\mathbb{Z}}{N \mathbb{Z}} \times \frac{\mathbb{R}^{2}}{\Gamma^{*}}\right)$, we have

$$
\frac{1}{C}\|m\|_{2}^{2} \leq I(m) \leq C\|m\|_{2}^{2}
$$

where

$$
I(m)=\int_{\mathbb{R}^{2}}\left|\sum_{j \in \mathbb{Z}} \sum_{k \in \frac{\mathbb{Z}}{N \mathbb{Z}}} 2^{j} m_{j, k}\left(2^{j} R^{k} \xi\right) \widehat{\psi}\left(2^{j} R^{k} \xi\right)\right|^{2} d \xi
$$


and

$$
\|m\|_{2}^{2}=\sum_{j \in \mathbb{Z}} \sum_{k \in \frac{\mathbb{Z}}{N \mathbb{Z}}} \int_{\frac{\mathbb{R}^{2}}{\Gamma^{*}}}\left|m_{j, k}(\xi)\right|^{2} d \xi
$$

As $\psi$ is bandlimited, it is quite straightforward to bound $I(m)$ above. Indeed, thanks to $\mathbf{H 3}$, most of the terms in the sum that defines $I(m)$ have disjoint supports. By developing the squared term, we have therefore

$$
I(m)=\sum_{j \in \mathbb{Z}} \sum_{k \in \frac{Z}{N \mathbb{Z}}} \sum_{j^{\prime}=j-1}^{j+1} \sum_{k^{\prime}=k-1}^{k+1} \int_{\mathbb{R}^{2}} 2^{j+j^{\prime}} m_{j, k}\left(2^{j} R^{k} \xi\right) \overline{m_{j^{\prime}, k^{\prime}}\left(2^{j^{\prime}} R^{k^{\prime}} \xi\right)} \widehat{\psi}\left(2^{j} R^{k} \xi\right) \overline{\widehat{\psi}\left(2^{j^{\prime}} R^{k^{\prime}} \xi\right)} d \xi
$$

The classical Young inequality states that, for all $a, b \in \mathbb{C}, a \bar{b}+\bar{a} b \leq|a|^{2}+|b|^{2}$. Thus, using in a row the changes of variables $\xi \mapsto 2^{j} R^{k} \xi$ and the fact that $m_{j, k}$ is $\gamma$-periodic for all $\gamma \in \Gamma^{*}$, we obtain

$$
\begin{aligned}
I(m) & \leq 9 \sum_{j \in \mathbb{Z}} \sum_{k \in \frac{Z}{N Z}} \int_{\mathbb{R}^{2}} 2^{2 j}\left|m_{j, k}\left(2^{j} R^{k} \xi\right) \widehat{\psi}\left(2^{j} R^{k} \xi\right)\right|^{2} d \xi \\
& =9 \sum_{j \in \mathbb{Z}} \sum_{k \in \frac{Z}{N \mathbb{Z}}} \int_{\mathbb{R}^{2}}\left|m_{j, k}(\xi) \widehat{\psi}(\xi)\right|^{2} d \xi \\
& =9 \sum_{j \in \mathbb{Z}} \sum_{k \in \frac{Z}{N \mathbb{Z}}} \int_{\frac{\mathbb{R}^{2}}{\Gamma^{*}}}\left|m_{j, k}(\xi)\right|^{2} \sum_{\gamma \in \Gamma^{*}}|\widehat{\psi}(\xi+\gamma)|^{2} d \xi
\end{aligned}
$$

which leads to

$$
I(m) \leq 9\left\|\sum_{\gamma \in \Gamma^{*}}|\widehat{\psi}(\cdot+\gamma)|^{2}\right\|_{\infty}\|m\|_{2}^{2}
$$

We have therefore the second inequality of (14).

Let us now bound $I(m)$ below. In order to simplify the notations, we make the substitutions $\xi \mapsto$ $2^{-j} R^{-k} \xi$ in each integral, and $j^{\prime} \mapsto j^{\prime}-j, k^{\prime} \mapsto k^{\prime}-k$ in sums. We get

$$
I(m)=\sum_{j \in \mathbb{Z}} \sum_{k \in \frac{Z}{N Z}} \sum_{j^{\prime}=-1}^{1} \sum_{k^{\prime}=-1}^{1} \int_{\mathbb{R}^{2}} 2^{j^{\prime}} m_{j, k}(\xi) \overline{m_{j^{\prime}, k^{\prime}}\left(2^{j^{\prime}} R^{k^{\prime}} \xi\right)} \widehat{\psi}(\xi) \overline{\widehat{\psi}\left(2^{j^{\prime}} R^{k^{\prime}} \xi\right)} d \xi
$$

Set therefore

$$
I(m)=\sum_{j \in \mathbb{Z}} \sum_{k \in \frac{\mathbb{Z}}{N \mathbb{Z}}} \int_{\frac{\mathbb{R}^{2}}{\Gamma^{*}}}\left|m_{j, k}(\xi)\right|^{2} \sum_{\gamma \in \Gamma^{*}}|\widehat{\psi}(\xi+\gamma)|^{2} d \xi+I_{1}(m)+I_{2}(m)+I_{3}(m)+I_{4}(m),
$$

where

$$
\begin{aligned}
& I_{1}(m)=4 \operatorname{Re}\left(\sum_{j \in \mathbb{Z}} \sum_{k \in \frac{\mathbb{Z}}{N \mathbb{Z}}} \int_{\mathbb{R}^{2}} m_{j, k}(\xi) \overline{m_{j+1, k}(2 \xi)} \widehat{\psi}(\xi) \overline{\widehat{\psi}(2 \xi)} d \xi\right) \\
& I_{2}(m)=2 \operatorname{Re}\left(\sum_{j \in \mathbb{Z}} \sum_{k \in \frac{\mathbb{Z}}{N \mathbb{Z}}} \int_{\mathbb{R}^{2}} m_{j, k}(\xi) \overline{m_{j, k+1}(R \xi)} \widehat{\psi}(\xi) \overline{\widehat{\psi}(R \xi)} d \xi\right) \\
& I_{3}(m)=4 \operatorname{Re}\left(\sum_{j \in \mathbb{Z}} \sum_{k \in \frac{\mathbb{Z}}{N \mathbb{Z}}} \int_{\mathbb{R}^{2}} m_{j, k}(\xi) \overline{m_{j+1, k+1}(2 R \xi)} \widehat{\psi}(\xi) \overline{\widehat{\psi}(2 R \xi)} d \xi\right) \\
& I_{4}(m)=4 \operatorname{Re}\left(\sum_{j \in \mathbb{Z}} \sum_{k \in \frac{\mathbb{Z}}{N \mathbb{Z}}} \int_{\mathbb{R}^{2}} m_{j, k}(\xi) \overline{m_{j+1, k-1}\left(2 R^{-1} \xi\right)} \widehat{\psi}(\xi) \overline{\hat{\psi}\left(2 R^{-1} \xi\right)} d \xi\right) .
\end{aligned}
$$


In order to bound $I(m)$ below, we can bound $I_{1}(m), I_{2}(m)$ and $I_{3}(m)+I_{4}(m)$ separately, which is the aim of the following lemma.

Lemma 3 For all $m \in L^{2}\left(\mathbb{Z} \times \frac{\mathbb{Z}}{N \mathbb{Z}} \times \frac{\mathbb{R}^{2}}{\Gamma^{*}}\right)$, we have

$$
\begin{array}{ll}
\text { (i) } \quad & \left|I_{1}(m)\right| \leq \int_{\frac{\mathbb{R}^{2}}{\Gamma^{*}}}\left(\sum_{j \in \mathbb{Z}} \sum_{k \in \frac{\mathbb{Z}}{N^{Z}}}\left|m_{j, k}(\xi)\right|^{2}\right) \Psi_{1}(\xi) d \xi, \\
\text { (ii }) \quad & \left|I_{2}(m)\right| \leq \int_{\frac{\mathbb{R}^{2}}{\Gamma^{*}}}\left(\sum_{j \in \mathbb{Z}} \sum_{k \in \frac{\mathbb{Z}}{N^{Z}}}\left|m_{j, k}(\xi)\right|^{2}\right) \Psi_{2}(\xi) d \xi, \\
\text { (iii }) & I_{3}(m)+I_{4}(m) \geq \int_{\frac{\mathbb{R}^{2}}{\Gamma^{*}}}\left(\sum_{j \in \mathbb{Z}} \sum_{k \in \frac{\mathbb{Z}}{N \mathbb{Z}}}\left|m_{j, k}(\xi)\right|^{2}\right)\left(\Psi_{4}(\xi)-\Psi_{3}(\xi)\right) d \xi,
\end{array}
$$

where $\Psi_{1}, \Psi_{2}, \Psi_{3}$ and $\Psi_{4}$ are given in (4), (5), (6) and (8), respectively.

We infer that

$$
I(m) \geq \int_{\frac{\mathbb{R}^{2}}{\Gamma^{*}}}\left(\sum_{j \in \mathbb{Z}} \sum_{k \in \frac{\mathbb{Z}}{N \mathbb{Z}}}\left|m_{j, k}(\xi)\right|^{2}\right)\left(\sum_{\gamma \in \Gamma^{*}}|\widehat{\psi}(\xi+\gamma)|^{2}-\Psi_{1}(\xi)-\Psi_{2}(\xi)-\Psi_{3}(\xi)+\Psi_{4}(\xi)\right) d \xi,
$$

where, by $\mathbf{H 4}$,

$$
\sum_{\gamma \in \Gamma^{*}}|\widehat{\psi}(\xi+\gamma)|^{2}-\Psi_{1}(\xi)-\Psi_{2}(\xi)-\Psi_{3}(\xi)+\Psi_{4}(\xi)>0, \quad \forall \xi \in \mathbb{R}^{2} .
$$

As the latter function is continuous and periodic, it is bounded below by a positive constant $C$ and we conclude that

$$
I(m) \geq C \int_{\frac{\mathbb{R}^{2}}{\Gamma^{*}}}\left(\sum_{j \in \mathbb{Z}} \sum_{k \in \frac{\mathbb{Z}}{N^{Z}}}\left|m_{j, k}(\xi)\right|^{2}\right) d \xi
$$

\subsection{Proof of Theorem 2}

Showing that the sequence $\left(\psi_{j, k, \gamma}\right)_{(j, k, \gamma) \in \mathbb{Z} \times \frac{Z}{N \mathbb{Z}} \times \Gamma}$ is global for $L^{2}\left(\mathbb{R}^{2}\right)$ amounts to show that

$$
\left(\operatorname{span}\left(\psi_{j, k, \gamma}\right)_{(j, k, \gamma) \in \mathbb{Z} \times \frac{\mathbb{Z}}{N \mathbb{Z}} \times \Gamma}\right)^{\perp}=\{0\} .
$$

Since, by H6, we have $\sum_{j \in \mathbb{Z}} \sum_{k \in \frac{Z}{N Z}}\left|\widehat{\psi}\left(2^{j} R^{k} \xi\right)\right|^{2}-\Phi_{1}(\xi)-\Phi_{2}(\xi)-\Phi_{3}(\xi)>0$, for almost every $\xi \in \mathbb{R}^{2}$, (20) is actually a straightforward consequence of the following inequality that we are going to prove: For all $f \in L^{2}\left(\mathbb{R}^{2}\right)$, we have

$$
\sum_{j \in \mathbb{Z}} \sum_{k \in \frac{\mathbb{Z}}{N \mathbb{Z}}} \sum_{\gamma \in \Gamma}\left|\left\langle f, \psi_{j, k, \gamma}\right\rangle\right|^{2} \geq \frac{\mathcal{L}(\mathcal{I})}{(2 \pi)^{4}} \int_{\mathbb{R}^{2}}|\hat{f}(\xi)|^{2}\left(\sum_{j \in \mathbb{Z}} \sum_{k \in \frac{\mathbb{Z}}{N \mathbb{Z}}}\left|\widehat{\psi}\left(2^{j} R^{k} \xi\right)\right|^{2}-\Phi_{1}(\xi)-\Phi_{2}(\xi)-\Phi_{3}(\xi)+\Phi_{4}(\xi)\right) d \xi .
$$

First, we apply Plancherel's theorem in order to show that, for all $j \in \mathbb{Z}, k \in \frac{\mathbb{Z}}{N \mathbb{Z}}, \gamma \in \Gamma$,

$$
\begin{aligned}
\left|\left\langle f, \psi_{j, k, \gamma}\right\rangle\right|^{2} & =\frac{1}{(2 \pi)^{4}}\left|\left\langle\hat{f}, \widehat{\psi_{j, k, \gamma}}\right\rangle\right|^{2} \\
& =\frac{1}{(2 \pi)^{4}}\left|\int_{\mathbb{R}^{2}} \hat{f}(\xi) 2^{-j} \overline{\widehat{\psi}\left(2^{-j} R^{k} \xi\right) e^{-i \gamma \cdot 2^{-j} R^{k} \xi}} d \xi\right|^{2}
\end{aligned}
$$




$$
\begin{aligned}
& =\frac{1}{(2 \pi)^{4}}\left|\int_{\mathbb{R}^{2}} 2^{j} \hat{f}\left(2^{j} R^{-k} \xi\right) \overline{\hat{\psi}(\xi)} e^{i \gamma \cdot \xi} d \xi\right|^{2} \\
& =\frac{1}{(2 \pi)^{4}} \mid \int_{\frac{\mathbb{R}^{2}}{\Gamma^{*}}} 2^{j} \sum_{\gamma^{\prime} \in \Gamma^{*}} \hat{f}\left(\left.2^{j} R^{-k}\left(\xi-\gamma^{\prime}\right) \overline{\hat{\psi}\left(\xi-\gamma^{\prime}\right)} e^{i \gamma \cdot \xi} d \xi\right|^{2}\right. \\
& =\frac{\mathcal{L}(\mathcal{I})}{(2 \pi)^{4}}\left|c_{-\gamma}\left(g_{j, k}\right)\right|^{2}
\end{aligned}
$$

where $g_{j, k}=\left(\xi \mapsto 2^{j} \sum_{\gamma \in \Gamma^{*}} \hat{f}\left(2^{j} R^{-k}(\xi-\gamma)\right) \overline{\widehat{\psi}(\xi-\gamma)}\right)$ and $c_{\gamma}(g)=\frac{1}{\mathcal{L}(\mathcal{I})} \int_{\frac{\mathbb{R}^{2}}{\Gamma^{*}}} g(x) e^{-i \gamma \cdot x} d x$ is the Fourier coefficient, of index $\gamma$, of a function $g$. Hypothesis $\mathbf{H 5}$ ensures that, for all $\xi \in \mathbb{R}^{2}, \widehat{\psi}(\xi-\gamma) \neq 0$ for only 4 values of $\gamma \in \Gamma^{*}$. We have therefore, for almost every $\xi \in \mathbb{R}^{2}$,

$$
\left|g_{j, k}(\xi)\right| \leq 4 \max _{\gamma \in \Gamma^{*}}\left|2^{j} \hat{f}\left(2^{j} R^{-k}(\xi-\gamma)\right) \widehat{\psi}(\xi-\gamma)\right| .
$$

Since $\hat{f} \in L^{2}\left(\mathbb{R}^{2}\right)$ and $\widehat{\psi} \in L^{\infty}\left(\mathbb{R}^{2}\right)$, we infer that $g_{j, k} \in L^{2}\left(\frac{\mathbb{R}^{2}}{\Gamma^{*}}\right)$. Thus, we can apply Parseval's theorem to $g_{j, k}$ and show that

$$
\begin{aligned}
& \sum_{\gamma \in \Gamma}\left|\left\langle f, \psi_{j, k, \gamma}\right\rangle\right|^{2}=\frac{\mathcal{L}(\mathcal{I})}{(2 \pi)^{4}} \sum_{\gamma \in \Gamma}\left|c_{\gamma}\left(g_{j, k}\right)\right|^{2}=\frac{\mathcal{L}(\mathcal{I})}{(2 \pi)^{4}} \int_{\frac{\mathbb{R}^{2}}{\Gamma^{*}}}\left|g_{j, k}(\xi)\right|^{2} d \xi \\
& =\frac{\mathcal{L}(\mathcal{I})}{(2 \pi)^{4}} \int_{\frac{\mathbb{R}^{2}}{\Gamma^{*}}}\left|\sum_{\gamma \in \Gamma^{*}} \hat{f}\left(\xi-2^{j} R^{-k} \gamma\right) \overline{\hat{\psi}\left(2^{j} R^{k} \xi-\gamma\right)}\right|^{2} d \xi \\
& =\frac{\mathcal{L}(\mathcal{I})}{(2 \pi)^{4}} \int_{\mathbb{R}^{2}} \hat{f}(\xi) \overline{\widehat{\psi}\left(2^{j} R^{k} \xi\right)}\left(\sum_{\gamma \in \Gamma^{*}} \overline{\hat{f}\left(\xi-2^{j} R^{-k} \gamma\right)} \widehat{\psi}\left(2^{j} R^{k} \xi-\gamma\right)\right) d \xi
\end{aligned}
$$

We deduce that

$$
\sum_{j \in \mathbb{Z}} \sum_{k \in \mathbb{Z}} \sum_{\gamma \in \Gamma}\left|\left\langle f, \psi_{j, k, \gamma}\right\rangle\right|^{2}=\frac{\mathcal{L}(\mathcal{I})}{(2 \pi)^{4}} \int_{\mathbb{R}^{2}}|\hat{f}(\xi)|^{2} \sum_{j \in \mathbb{Z}} \sum_{k \in \frac{\mathbb{Z}}{N Z}}\left|\widehat{\psi}\left(2^{j} R^{-k} \xi\right)\right|^{2} d \xi+\frac{\mathcal{L}(\mathcal{I})}{(2 \pi)^{4}} \sum_{\gamma \in G} J_{\gamma},
$$

where

$$
J_{\gamma}=\sum_{j \in \mathbb{Z}} \sum_{k \in \frac{\mathbb{Z}}{N \mathbb{Z}}} \int_{\mathbb{R}^{2}} \hat{f}(\xi) \overline{\widehat{\psi}\left(2^{-j} R^{k} \xi\right) \hat{f}\left(\xi-2^{j} R^{-k} \gamma\right)} \widehat{\psi}\left(2^{-j} R^{k} \xi-\gamma\right) d \xi
$$

and, thanks to $\mathbf{H} \mathbf{5}$ again,

$$
G=\{\mu,-\mu, 2 \mu,-2 \mu, 3 \eta,-3 \eta, 3 \tilde{\eta},-3 \tilde{\eta}, \nu,-\nu, \tilde{\nu},-\tilde{\nu}\} .
$$

However, using a change of variable, we show that, for all $\gamma \in G$,

$$
J_{\gamma}=\sum_{j \in \mathbb{Z}} \sum_{k \in \frac{\mathbb{Z}}{N \mathbb{Z}}} \int_{\mathbb{R}^{2}} \hat{f}\left(\xi+2^{j} R^{-k} \gamma\right) \overline{\hat{\psi}\left(2^{-j} R^{k} \xi+\gamma\right) \hat{f}(\xi)} \widehat{\psi}\left(2^{-j} R^{k} \xi\right) d \xi=\overline{J_{-\gamma}} .
$$

We have therefore $J_{\gamma}+J_{-\gamma}=2 \operatorname{Re}\left(J_{\gamma}\right)$. Putting this all together with the next lemma, we see that (21) is satisfied.

Lemma 4 For all $f \in L^{2}\left(\mathbb{R}^{2}\right)$, we have

$$
\begin{aligned}
\text { (i) } \quad & 2\left|J_{\mu}+J_{2 \mu}\right| \leq \int_{\mathbb{R}^{2}}|\hat{f}(\xi)|^{2} \Phi_{1}(\xi) d \xi, \\
\text { (ii) } \quad & 2\left|J_{3 \eta}+J_{3 \tilde{\eta}}\right| \leq \int_{\mathbb{R}^{2}}|\hat{f}(\xi)|^{2} \Phi_{2}(\xi) d \xi, \\
\text { (iii) } \quad & 2 \operatorname{Re}\left(J_{\nu}+J_{\tilde{\nu}}\right) \geq \int_{\mathbb{R}^{2}}|\hat{f}(\xi)|^{2}\left(\Phi_{4}(\xi)-\Phi_{3}(\xi)\right) d \xi,
\end{aligned}
$$


where $\Phi_{1}, \Phi_{2}, \Phi_{3}$ and $\Phi_{4}$ are given in (10),(11),(12) and (13), respectively.

If we assume now that the inequality (9) in $\mathbf{H 6}$ is satisfied for all $\xi \in \mathbb{R}^{2}$, then the function

$$
\xi \mapsto \frac{\mathcal{L}(\mathcal{I})}{(2 \pi)^{4}}\left(\sum_{j \in \mathbb{Z}} \sum_{k \in \frac{\mathbb{Z}}{N Z}}\left|\widehat{\psi}\left(2^{j} R^{k} \xi\right)\right|^{2}-\Phi_{1}(\xi)-\Phi_{2}(\xi)-\Phi_{3}(\xi)+\Phi_{4}(\xi)\right)
$$

is bounded below by a positive constant that we denote by $1 / C$. Indeed, since it is invariant by dilation of factor 2 , its minimum value can be calculated for $\xi$ in a ring where all the sums are finite. We have therefore

$$
\sum_{j \in \mathbb{Z}} \sum_{k \in \mathbb{Z}} \sum_{\gamma \in \Gamma}\left|\left\langle f, \psi_{j, k, \gamma}\right\rangle\right|^{2} \geq \frac{1}{C} \int_{\mathbb{R}^{2}}|\hat{f}(\xi)|^{2} d \xi
$$

On the other hand, one can easily show that $2\left|J_{\nu}+J_{\tilde{\nu}}\right| \leq \int_{\mathbb{R}^{2}}|\hat{f}(\xi)|^{2} \Phi_{3}(\xi) d \xi$ - see the proof of (iii). Hence

$$
\sum_{j \in \mathbb{Z}} \sum_{k \in \frac{\mathbb{Z}}{N \mathbb{Z}}} \sum_{\gamma \in \Gamma}\left|\left\langle f, \psi_{j, k, \gamma}\right\rangle\right|^{2} \leq \frac{\mathcal{L}(\mathcal{I})}{(2 \pi)^{4}} \int_{\mathbb{R}^{2}}|\hat{f}(\xi)|^{2}\left(\sum_{j \in \mathbb{Z}} \sum_{k \in \frac{\mathbb{Z}}{N \mathbb{Z}}}\left|\widehat{\psi}\left(2^{j} R^{k} \xi\right)\right|^{2}+\Phi_{1}(\xi)+\Phi_{2}(\xi)+\Phi_{3}(\xi)\right) d \xi .
$$

We infer that, even if it means increasing the value of $C$, we have

$$
\sum_{j \in \mathbb{Z}} \sum_{k \in \frac{Z}{N \mathbb{Z}}} \sum_{\gamma \in \Gamma}\left|\left\langle f, \psi_{j, k, \gamma}\right\rangle\right|^{2} \leq C \int_{\mathbb{R}^{2}}|\hat{f}(\xi)|^{2} d \xi
$$

and we conclude that $\left(\psi_{j, k, \gamma}\right)_{j \in \mathbb{Z}, k \in \mathbb{Z}, \gamma \in \Gamma}$ is a frame for $L^{2}\left(\mathbb{R}^{2}\right)$.

\subsection{Proof of lemmas}

\subsubsection{Proof of Lemma 1}

The two identities can be proven by straightforward calculation using (1). First, we have

$$
\begin{aligned}
\sum_{\gamma \in \Gamma^{*}}|\widehat{\psi}(\xi-\gamma)|^{2} & =\sum_{k \in \mathbb{Z}^{2}}\left|\widehat{\psi}\left(\xi-k_{1} \mu-k_{2} \nu\right)\right|^{2} \\
& =\sum_{k \in \mathbb{Z}^{2}}\left|\widehat{\psi_{a}}\left(\xi-k_{1} \mu-k_{2} \nu\right)\right|^{2}+\sum_{k \in \mathbb{Z}^{2}}\left|\widehat{\psi_{a a}}\left(\xi-k_{1} \mu-k_{2} \nu\right)\right|^{2} \\
& =\sum_{k \in \mathbb{Z}^{2}}\left(\left|\widehat{\psi_{a}}\left(\xi-k_{1} \mu-k_{2} \nu\right)\right|^{2}+\left|\widehat{\psi_{a a}}\left(\xi-\left(k_{1}+1\right) \mu-\left(k_{2}+1\right) \nu\right)\right|^{2}\right) \\
& =\mathcal{L}(\mathcal{I})^{-1} \sum_{k \in \mathbb{Z}}\left|H_{\delta}\left(\xi_{1}-(k-1) \pi\right)\right|^{2}\left|H_{\delta}\left((2-k) \pi-\xi_{1}\right)\right|^{2}=\mathcal{L}(\mathcal{I})^{-1}
\end{aligned}
$$

In order to prove the second identity, let us denote by $R^{\alpha}$ the rotation of angle $\alpha \frac{(N-1) \pi}{N}$. This allows for writing

$$
V_{\tau}(\xi)=H_{\tau}\left(\left(R^{\frac{1}{2}} \xi\right)_{1}\right) H_{\tau}\left(\left(R^{-\frac{1}{2}} \xi\right)_{1}\right)+H_{\tau}\left(-\left(R^{\frac{1}{2}} \xi\right)_{1}\right) H_{\tau}\left(-\left(R^{-\frac{1}{2}} \xi\right)_{1}\right), \quad \forall \xi \in \mathbb{R}^{2} .
$$

We have therefore

$$
\begin{aligned}
\left|\widehat{\psi}_{a}(\xi)\right| & =2 \pi(\mathcal{L}(\mathcal{I}))^{-\frac{1}{2}} H_{\delta}\left(\xi_{1}-\pi\right) H_{\delta}\left(2 \pi-\xi_{1}\right) V_{\tau}(\xi) \\
& =2 \pi(\mathcal{L}(\mathcal{I}))^{-\frac{1}{2}} H_{\delta}\left(\xi_{1}-\pi\right) H_{\delta}\left(2 \pi-\xi_{1}\right) H_{\tau}\left(\left(R^{\frac{1}{2}} \xi\right)_{1}\right) H_{\tau}\left(\left(R^{-\frac{1}{2}} \xi\right)_{1}\right) .
\end{aligned}
$$


On the other hand, we have

$$
\begin{aligned}
\left|\widehat{\psi}_{a a}(R \xi)\right| & =2 \pi(\mathcal{L}(\mathcal{I}))^{-\frac{1}{2}} H_{\delta}\left(-(R \xi)_{1}-\pi\right) H_{\delta}\left((R \xi)_{1}+2 \pi\right) V_{\tau}(R \xi) \\
& =2 \pi(\mathcal{L}(\mathcal{I}))^{-\frac{1}{2}} H_{\delta}\left(-(R \xi)_{1}-\pi\right) H_{\delta}\left((R \xi)_{1}+2 \pi\right) H_{\tau}\left(-\left(R^{\frac{1}{2}} R \xi\right)_{1}\right) H_{\tau}\left(-\left(R^{-\frac{1}{2}} R \xi\right)_{1}\right) \\
& =2 \pi(\mathcal{L}(\mathcal{I}))^{-\frac{1}{2}} H_{\delta}\left(-(R \xi)_{1}-\pi\right) H_{\delta}\left((R \xi)_{1}+2 \pi\right) H_{\tau}\left(-\left(R^{\frac{3}{2}} \xi\right)_{1}\right) H_{\tau}\left(-\left(R^{\frac{1}{2}} \xi\right)_{1}\right) .
\end{aligned}
$$

Since $\tau \leq(\pi-\delta) \sin \left(\frac{\pi}{2 N}\right)$, we can check easily that $H_{\tau}\left(-\left(R^{\frac{3}{2}} \xi\right)_{1}\right)=1$, for all $\xi \in \operatorname{supp} \widehat{\psi}_{a}$, and $H_{\tau}\left(\left(R^{-\frac{1}{2}} \xi\right)_{1}\right)=1$, for all $\xi \in \operatorname{supp} \widehat{\psi}_{a a} \circ R$. We deduce

$\left|\widehat{\psi}_{a a}(R \xi) \widehat{\psi}_{a}(\xi)\right|=\frac{(2 \pi)^{2}}{\mathcal{L}(\mathcal{I})} H_{\delta}\left(-(R \xi)_{1}-\pi\right) H_{\delta}\left((R \xi)_{1}+2 \pi\right) H_{\tau}\left(-\left(R^{\frac{1}{2}} \xi\right)_{1}\right) H_{\delta}\left(\xi_{1}-\pi\right) H_{\delta}\left(2 \pi-\xi_{1}\right) H_{\tau}\left(\left(R^{\frac{1}{2}} \xi\right)_{1}\right)$.

As $\left|\widehat{\psi}_{a a}(\xi)\right|=\left|\widehat{\psi}_{a}(-\xi)\right|$, we have also

$\left|\widehat{\psi}_{a}(R \xi) \widehat{\psi}_{a a}(\xi)\right|=\frac{(2 \pi)^{2}}{\mathcal{L}(\mathcal{I})} H_{\delta}\left((R \xi)_{1}-\pi\right) H_{\delta}\left(2 \pi-(R \xi)_{1}\right) H_{\tau}\left(\left(R^{\frac{1}{2}} \xi\right)_{1}\right) H_{\delta}\left(-\xi_{1}-\pi\right) H_{\delta}\left(2 \pi+\xi_{1}\right) H_{\tau}\left(-\left(R^{\frac{1}{2}} \xi\right)_{1}\right)$

that can be re-ordered into

$\left|\widehat{\psi}_{a}(R \xi) \widehat{\psi}_{a a}(\xi)\right|=\frac{(2 \pi)^{2}}{\mathcal{L}(\mathcal{I})} H_{\delta}\left(2 \pi-(R \xi)_{1}\right) H_{\delta}\left((R \xi)_{1}-\pi\right) H_{\tau}\left(-\left(R^{\frac{1}{2}} \xi\right)_{1}\right) H_{\delta}\left(2 \pi+\xi_{1}\right) H_{\delta}\left(-\xi_{1}-\pi\right) H_{\tau}\left(\left(R^{\frac{1}{2}} \xi\right)_{1}\right)$.

Using the fact that $(3 \eta)_{1}=3 \pi=-(3 \tilde{\eta})_{1}=-(R(3 \eta))_{1}$ and $\left(R^{\frac{1}{2}}(3 \eta)\right)_{1}=0$, we conclude that

$$
\left|\widehat{\psi}_{a}(R(\xi-3 \eta)) \widehat{\psi}_{a a}(\xi-3 \eta)\right|=\left|\widehat{\psi}_{a a}(R \xi) \widehat{\psi}_{a}(\xi)\right|
$$

\subsubsection{Proof of Lemma 2}

By definition, $\left(\psi_{j, k, \gamma}\right)_{j \in \mathbb{Z}, k \in \frac{\mathbb{Z}}{N \mathbb{Z}}, \gamma \in \Gamma}$ is a Riesz sequence is and only if there exist $C \geq 1$ such that, for all $a \in \ell^{2}\left(\mathbb{Z} \times \frac{\mathbb{Z}}{N \mathbb{Z}} \times \Gamma\right)$,

$$
\frac{1}{C}\|a\|_{2} \leq\left\|\sum_{j \in \mathbb{Z}} \sum_{k \in \frac{\mathbb{Z}}{N \mathbb{Z}}} \sum_{\gamma \in \Gamma} a_{j, k, \gamma} \psi_{j, k, \gamma}\right\|_{2} \leq C\|a\|_{2}
$$

or, in other words,

$$
\frac{1}{C^{2}} \sum_{j \in \mathbb{Z}} \sum_{k \in \frac{\mathbb{Z}}{N \mathbb{Z}}} \sum_{\gamma \in \Gamma}\left|a_{j, k, \gamma}\right|^{2} \leq \int_{\mathbb{R}^{2}}\left|\sum_{j \in \mathbb{Z}} \sum_{k \in \frac{\mathbb{Z}}{N \mathbb{Z}}} \sum_{\gamma \in \Gamma} a_{j, k, \gamma} 2^{j} \psi\left(2^{j} R^{k} x-\gamma\right)\right|^{2} d x \leq C^{2} \sum_{j \in \mathbb{Z}} \sum_{k \in \frac{\mathbb{Z}}{N \mathbb{Z}}} \sum_{\gamma \in \Gamma}\left|a_{j, k, \gamma}\right|^{2} .
$$

By Plancherel's theorem and putting $m=\left(m_{j, k}\right)_{(j, k) \in \mathbb{Z} \times \frac{\mathbb{Z}}{N \mathbb{Z}}}$ where $m_{j, k}(\xi)=\sum_{\gamma \in \Gamma} a_{j, k, \gamma} e^{-i \gamma \cdot \xi}$, it amounts to show that there exist $C \geq 1$ such that, for all $m \in L^{2}\left(\mathbb{Z} \times \frac{\mathbb{Z}}{N \mathbb{Z}} \times \frac{\mathbb{R}^{2}}{\Gamma^{*}}\right)$,

$$
\frac{1}{C}\|m\|_{2}^{2} \leq \int_{\mathbb{R}^{2}}\left|\sum_{j \in \mathbb{Z}} \sum_{k \in \frac{\mathbb{Z}}{N \mathbb{Z}}} 2^{j} m_{j, k}\left(2^{j} R^{k} \xi\right) \widehat{\psi}\left(2^{j} R^{k} \xi\right)\right|^{2} d \xi \leq C\|m\|_{2}^{2},
$$

where

$$
\|m\|_{2}^{2}=\sum_{j \in \mathbb{Z}} \sum_{k \in \frac{\mathbb{Z}}{N \mathbb{Z}}} \int_{\frac{\mathbb{R}^{2}}{\Gamma^{*}}}\left|m_{j, k}(\xi)\right|^{2} d \xi
$$




\subsubsection{Proof of Lemma 3-(i)}

First, we develop $\widehat{\psi}$ as $\widehat{\psi}=\widehat{\psi}_{a}+\widehat{\psi}_{a a}$. By H3, we have $\widehat{\psi}_{a}(\cdot) \overline{\widehat{\psi}_{a a}(2 \cdot)}=0$ and $\widehat{\psi}_{a a}(\cdot) \overline{\widehat{\psi}_{a}(2 \cdot)}=0$. We deduce that $\widehat{\psi}(\cdot) \overline{\hat{\psi}(2 \cdot)}=\widehat{\psi}_{a}(\cdot) \overline{\widehat{\psi}_{a}(2 \cdot)}+\widehat{\psi}_{a a}(\cdot) \overline{\widehat{\psi}_{a a}(2 \cdot)}$ and

$$
\begin{aligned}
I_{1} & =4 \operatorname{Re}\left(\sum_{j \in \mathbb{Z}} \sum_{k \in \mathbb{Z}} \int_{\mathbb{R}^{2}} m_{j, k}(\xi) \overline{m_{j+1, k}(2 \xi)}\left(\widehat{\psi}_{a}(\xi) \overline{\widehat{\psi}_{a}(2 \xi)}+\widehat{\psi}_{a a}(\xi) \overline{\widehat{\psi}_{a a}(2 \xi)}\right) d \xi\right) \\
& =4 \sum_{j \in \mathbb{Z}} \sum_{k \in \mathbb{Z}} \operatorname{Re}\left(\int_{\mathbb{R}^{2}} m_{j, k}(\xi) \overline{m_{j+1, k}(2 \xi)} \widehat{\psi}_{a}(\xi) \overline{\widehat{\psi}_{a}(2 \xi)} d \xi+\int_{\mathbb{R}^{2}} m_{j, k}(\xi) \overline{m_{j+1, k}(2 \xi)} \widehat{\psi}_{a a}(\xi) \overline{\widehat{\psi}_{a a}(2 \xi)} d \xi\right) .
\end{aligned}
$$

Note that $m_{j, k}$ and $m_{j+1, k}(2 \cdot)$ are $\mu$-periodic. Making the substitution of variables $\xi \mapsto \xi-\mu$ in the second integral, we have therefore

$$
I_{1}=4 \sum_{j \in \mathbb{Z}} \sum_{k \in \frac{Z}{N Z}} \operatorname{Re}\left(\int_{\mathbb{R}^{2}} m_{j, k}(\xi) \overline{m_{j+1, k}(2 \xi)}\left(\widehat{\psi}_{a}(\xi) \overline{\widehat{\psi}_{a}(2 \xi)}+\widehat{\psi}_{a a}(\xi-\mu) \overline{\widehat{\psi}_{a a}(2 \xi-2 \mu)}\right) d \xi\right) .
$$

By using $\widehat{\psi}(\xi)=e^{\frac{i \xi_{1}}{2}}|\widehat{\psi}(\xi)|$ and $e^{\frac{i \mu_{1}}{2}}=e^{i \pi}=-1$, we show next that

$$
\begin{aligned}
\left|\widehat{\psi}_{a}(\xi) \overline{\widehat{\psi}_{a}(2 \xi)}+\widehat{\psi}_{a a}(\xi-\mu) \overline{\widehat{\psi}_{a a}(2 \xi-2 \mu)}\right| & =\left|e^{\frac{-i \xi_{1}}{2}}\right| \widehat{\psi}_{a}(\xi) \widehat{\psi}_{a}(2 \xi)\left|+e^{\frac{-i\left(\xi_{1}+\mu_{1}\right)}{2}}\right| \widehat{\psi}_{a a}(\xi-\mu) \widehat{\psi}_{a a}(2 \xi-2 \mu)|| \\
& =|| \widehat{\psi}_{a}(\xi) \widehat{\psi}_{a}(2 \xi)|-| \widehat{\psi}_{a a}(\xi-\mu) \widehat{\psi}_{a a}(2 \xi-2 \mu) \mid .
\end{aligned}
$$

Thus, we obtain the following bound for $I_{1}$.

$$
\left|I_{1}\right| \leq 4 \sum_{j \in \mathbb{Z}} \sum_{k \in \mathbb{Z}} \int_{\mathbb{R}^{2}}\left|m_{j, k}(\xi) m_{j+1, k}(2 \xi)\right||| \widehat{\psi}_{a}(\xi) \widehat{\psi}_{a}(2 \xi)|-| \widehat{\psi}_{a a}(\xi-\mu) \widehat{\psi}_{a a}(2 \xi-2 \mu)|| d \xi .
$$

¿From Young inequality, we deduce that

$$
\left|I_{1}\right| \leq \sum_{j \in \mathbb{Z}} \sum_{k \in \frac{Z}{N Z}} \int_{\mathbb{R}^{2}}\left(\left|m_{j, k}(\xi)\right|^{2}+4\left|m_{j+1, k}(2 \xi)\right|^{2}\right)|| \widehat{\psi}_{a}(\xi) \widehat{\psi}_{a}(2 \xi)|-| \widehat{\psi}_{a a}(\xi-\mu) \widehat{\psi}_{a a}(2 \xi-2 \mu)|| d \xi
$$

Then we conclude by making another substitution of variables

$$
\begin{aligned}
\left|I_{1}\right| \leq & \sum_{j \in \mathbb{Z}} \sum_{k \in \in \mathbb{Z}} \int_{\mathbb{R}^{2}}\left|m_{j, k}(\xi)\right|^{2}|| \widehat{\psi}_{a}(\xi) \widehat{\psi}_{a}(2 \xi)|-| \widehat{\psi}_{a a}(\xi-\mu) \widehat{\psi}_{a a}(2 \xi-2 \mu)|| d \xi \\
& +\sum_{j \in \mathbb{Z}} \sum_{k \in \frac{\mathbb{Z}}{N \mathbb{Z}}} \int_{\mathbb{R}^{2}}\left|m_{j, k}(\xi)\right|^{2}|| \widehat{\psi}_{a}\left(\frac{\xi}{2}\right) \widehat{\psi}_{a}(\xi)|-| \widehat{\psi}_{a a}\left(\frac{\xi-\mu}{2}\right) \widehat{\psi}_{a a}(\xi-\mu)|| d \xi \\
= & \sum_{j \in \mathbb{Z}} \sum_{k \in \frac{\mathbb{Z}}{N \mathbb{Z}}} \int_{\frac{\mathbb{R}^{2}}{\Gamma^{*}}}\left|m_{j, k}(\xi)\right|^{2} \Psi_{1}(\xi) d \xi .
\end{aligned}
$$

\subsubsection{Proof of Lemma 3-(ii)}

The proof uses similar arguments to those used for $I_{1}$. By H3, we have $\widehat{\psi}(\cdot) \overline{\widehat{\psi}(R \cdot)}=\widehat{\psi}_{a}(\cdot) \overline{\widehat{\psi}_{a a}(R \cdot)}+$ $\widehat{\psi}_{a a}(\cdot) \overline{\widehat{\psi}_{a}(R \cdot)}$. Since $3 \eta \in \Gamma^{*}$ and $3 R \eta=3 \tilde{\eta} \in \Gamma^{*}$, the functions $m_{j, k}$ and $m_{j, k+1}(R \cdot)$ are $3 \eta$-periodic and we deduce

$$
\begin{aligned}
I_{2} & =2 \operatorname{Re}\left(\sum_{j \in \mathbb{Z}} \sum_{k \in \frac{Z}{N \mathbb{Z}}} \int_{\mathbb{R}^{2}} m_{j, k}(\xi) \overline{m_{j, k+1}(R \xi)}\left(\widehat{\psi}_{a}(\xi) \overline{\widehat{\psi}_{a a}(R \xi)}+\widehat{\psi}_{a a}(\xi) \overline{\widehat{\psi}_{a}(R \xi)}\right) d \xi\right) \\
& =2 \operatorname{Re}\left(\sum_{j \in \mathbb{Z}} \sum_{k \in \frac{Z}{N \mathbb{Z}}} \int_{\mathbb{R}^{2}} m_{j, k}(\xi) \overline{m_{j, k+1}(R \xi)}\left(\widehat{\psi}_{a}(\xi) \overline{\widehat{\psi}_{a a}(R \xi)}+\widehat{\psi}_{a a}(\xi-3 \eta) \overline{\widehat{\psi}_{a}(R \xi-3 R \eta)}\right) d \xi\right) .
\end{aligned}
$$


By using $\widehat{\psi}(\xi)=e^{\frac{i \xi_{1}}{2}}|\widehat{\psi}(\xi)|$ and $e^{\frac{i\left(3 \eta_{1}-3(R \eta)_{1}\right)}{2}}=e^{i 3 \pi}=-1$, we show next that

$$
\left|\widehat{\psi}_{a}(\xi) \overline{\widehat{\psi}_{a a}(R \xi)}+\widehat{\psi}_{a a}(\xi-3 \eta) \overline{\widehat{\psi}_{a}(R \xi-3 R \eta)}\right|=\left|\widehat{\psi}_{a}(\xi) \widehat{\psi}_{a a}(R \xi)\right|-\left|\widehat{\psi}_{a a}(\xi-3 \eta) \widehat{\psi}_{a}(R \xi-3 R \eta)\right| .
$$

We obtain therefore the following bound for $I_{2}$. Justifications are the same as in the proof of (i).

$$
\begin{aligned}
\left|I_{2}\right| \leq & 2 \sum_{j \in \mathbb{Z}} \sum_{k \in \frac{Z}{N \mathbb{Z}}} \int_{\mathbb{R}^{2}}\left|m_{j, k}(\xi) m_{j, k+1}(R \xi)\right|\left|\widehat{\psi}_{a}(\xi) \widehat{\psi}_{a a}(R \xi)\right|-\left|\widehat{\psi}_{a a}(\xi-3 \eta) \widehat{\psi}_{a}(R \xi-3 R \eta)\right| \mid d \xi \\
\leq & \sum_{j \in \mathbb{Z}} \sum_{k \in \frac{Z}{N Z}} \int_{\mathbb{R}^{2}}\left(\left|m_{j, k}(\xi)\right|^{2}+\left|m_{j, k+1}(R \xi)\right|^{2}\right)|| \widehat{\psi}_{a}(\xi) \widehat{\psi}_{a a}(R \xi)|-| \widehat{\psi}_{a a}(\xi-3 \eta) \widehat{\psi}_{a}(R \xi-3 R \eta) \mid d \xi \\
= & \sum_{j \in \mathbb{Z}} \sum_{k \in \frac{Z}{N Z}} \int_{\mathbb{R}^{2}}\left|m_{j, k}(\xi)\right|^{2}|| \widehat{\psi}_{a}(\xi) \widehat{\psi}_{a a}(R \xi)|-| \widehat{\psi}_{a a}(\xi-3 \eta) \widehat{\psi}_{a}(R \xi-3 R \eta)|| d \xi \\
& +\sum_{j \in \mathbb{Z}} \sum_{k \in \frac{\mathbb{Z}}{N \mathbb{Z}}} \int_{\mathbb{R}^{2}}\left|m_{j, k}(\xi)\right|^{2}|| \widehat{\psi}_{a}\left(R^{-1} \xi\right) \widehat{\psi}_{a a}(\xi)|-| \widehat{\psi}_{a a}\left(R^{-1} \xi-3 \eta\right) \widehat{\psi}_{a}(\xi-3 R \eta) \mid d \xi \\
= & \sum_{j \in \mathbb{Z}} \sum_{k \in \frac{\mathbb{Z}}{N \mathbb{Z}}} \int_{\frac{\mathbb{R}^{2}}{\Gamma^{*}}}\left|m_{j, k}(\xi)\right|^{2} \Psi_{2}(\xi) d \xi .
\end{aligned}
$$

\subsubsection{Proof of Lemma 3-(iii)}

Using H3 again, we see that $\widehat{\psi}_{a}(\cdot) \overline{\widehat{\psi}_{a}(2 R \cdot)}=\widehat{\psi}_{a a}(\cdot) \overline{\widehat{\psi}_{a a}(2 R \cdot)}=\widehat{\psi}_{a}(\cdot) \overline{\widehat{\psi}_{a}\left(2 R^{-1} \cdot\right)}=\widehat{\psi}_{a a}(\cdot) \overline{\widehat{\psi}_{a a}\left(R^{-1} \cdot\right)}=0$. We deduce

$$
\begin{aligned}
I_{3}+I_{4}= & 4 \sum_{j \in \mathbb{Z}} \operatorname{Re}\left(\int_{\mathbb{R}^{2}} \sum_{k \in \frac{\mathbb{Z}}{N \mathbb{Z}}} m_{j, k}(\xi) \overline{m_{j+1, k+1}(2 R \xi)} \widehat{\psi}_{a}(\xi) \overline{\widehat{\psi}_{a a}(2 R \xi)} d \xi\right. \\
& +\int_{\mathbb{R}^{2}} \sum_{k \in \frac{\mathbb{Z}}{N Z}} m_{j, k}(\xi) \overline{m_{j+1, k+1}(2 R \xi)} \widehat{\psi}_{a a}(\xi) \overline{\widehat{\psi}_{a}(2 R \xi)} d \xi \\
& +\int_{\mathbb{R}^{2}} \sum_{k \in \frac{\mathbb{Z}}{N \mathbb{Z}}} m_{j, k}(\xi) \overline{m_{j+1, k-1}\left(2 R^{-1} \xi\right)} \widehat{\psi}_{a a}(\xi) \overline{\widehat{\psi}_{a}\left(2 R^{-1} \xi\right)} d \xi \\
& \left.+\int_{\mathbb{R}^{2}} \sum_{k \in \frac{\mathbb{Z}}{N \mathbb{Z}}} m_{j, k}(\xi) \overline{m_{j+1, k-1}\left(2 R^{-1} \xi\right)} \widehat{\psi}_{a}(\xi) \overline{\widehat{\psi}_{a a}\left(2 R^{-1} \xi\right)} d \xi\right) \\
= & \sum_{j \in \mathbb{Z}} \operatorname{Re}\left(I_{5, j}+I_{6, j}\right),
\end{aligned}
$$

where

$$
\begin{aligned}
I_{5, j}= & 4 \sum_{k \in \frac{Z}{N Z}} \int_{\mathbb{R}^{2}} m_{j, k}(\xi) \overline{m_{j+1, k+1}(2 R \xi)} \widehat{\psi}_{a}(\xi) \overline{\widehat{\psi}_{a a}(2 R \xi)} d \xi \\
& +4 \sum_{k \in \frac{Z}{N Z}} \int_{\mathbb{R}^{2}} m_{j, k}(\xi) \overline{m_{j+1, k-1}\left(2 R^{-1} \xi\right)} \widehat{\psi}_{a a}(\xi) \overline{\widehat{\psi}_{a}\left(2 R^{-1} \xi\right)} d \xi
\end{aligned}
$$

and

$$
\begin{aligned}
I_{6, j}= & 4 \sum_{k \in \frac{Z}{N Z}} \int_{\mathbb{R}^{2}} m_{j, k}(\xi) \overline{m_{j+1, k+1}(2 R \xi)} \widehat{\psi}_{a a}(\xi) \overline{\widehat{\psi}_{a}(2 R \xi)} d \xi \\
& +4 \sum_{k \in \frac{Z}{N \mathbb{Z}}} \int_{\mathbb{R}^{2}} m_{j, k}(\xi) \overline{m_{j+1, k-1}\left(2 R^{-1} \xi\right)} \widehat{\psi}_{a}(\xi) \overline{\widehat{\psi}_{a a}\left(2 R^{-1} \xi\right)} d \xi .
\end{aligned}
$$


Let us focus on $I_{5, j}$. In the integrals of the first sum in $I_{5, j}$, we make the substitutions of variables $\xi \mapsto \eta+R^{k} \xi$, while in the second one, we apply $\xi \mapsto R \eta+R^{k} \xi$. We get

$$
\begin{aligned}
I_{5, j}= & 4 \sum_{k \in \frac{\mathbb{Z}}{N Z}} \int_{\mathbb{R}^{2}} m_{j, k}\left(\eta+R^{k} \xi\right) \overline{m_{j+1, k+1}\left(2 R \eta+2 R^{k+1} \xi\right)} \widehat{\psi}_{a}\left(\eta+R^{k} \xi\right) \overline{\widehat{\psi}_{a a}\left(2 R \eta+2 R^{k+1} \xi\right)} d \xi \\
& +4 \sum_{k \in \frac{\mathbb{Z}}{N Z}} \int_{\mathbb{R}^{2}} m_{j, k}\left(R \eta+R^{k} \xi\right) \overline{m_{j+1, k-1}\left(2 \eta+2 R^{k-1} \xi\right)} \widehat{\psi}_{a a}\left(R \eta+R^{k} \xi\right) \overline{\widehat{\psi}_{a}\left(2 \eta+2 R^{k-1} \xi\right)} d \xi
\end{aligned}
$$

Since $\eta-R \eta=\eta-\tilde{\eta}=\mu \in \Gamma^{*}$, we have $m_{j, k}(R \eta+\cdot)=m_{j, k}(\eta+\cdot)$ and $m_{j, k}(2 R \eta+\cdot)=m_{j, k}(2 \eta+\cdot)$. It follows that

$$
\begin{aligned}
I_{5, j}= & 4 \sum_{k \in \frac{Z}{N Z}} \int_{\mathbb{R}^{2}} m_{j, k}\left(\eta+R^{k} \xi\right)\left(\overline{m_{j+1, k+1}\left(2 \eta+2 R^{k+1} \xi\right)} \widehat{\psi}_{a}\left(\eta+R^{k} \xi\right) \overline{\widehat{\psi}_{a a}\left(2 \tilde{\eta}+2 R^{k+1} \xi\right)}\right. \\
& \left.+\overline{m_{j+1, k-1}\left(2 \eta+2 R^{k-1} \xi\right)} \widehat{\psi}_{a a}\left(\tilde{\eta}+R^{k} \xi\right) \overline{\widehat{\psi}_{a}\left(2 \eta+2 R^{k-1} \xi\right)}\right) d \xi .
\end{aligned}
$$

We use next $\mathbf{H 2}$ to show that

$$
\widehat{\psi}_{a}\left(\eta+R^{k} \xi\right) \overline{\widehat{\psi}_{a a}\left(2 \tilde{\eta}+2 R^{k+1} \xi\right)}=e^{\frac{i\left(\eta_{1}+\left(R^{k} \xi\right)_{1}\right)}{2}} \overline{e^{i\left(\tilde{\eta}_{1}+\left(R^{k+1} \xi\right)_{1}\right)}}\left|\widehat{\psi}_{a}\left(\eta+R^{k} \xi\right) \widehat{\psi}_{a a}\left(2 \tilde{\eta}+2 R^{k+1} \xi\right)\right|
$$

and

$$
\widehat{\psi}_{a a}\left(\tilde{\eta}+R^{k} \xi\right) \overline{\widehat{\psi}_{a}\left(2 \eta+2 R^{k-1} \xi\right)}=e^{\frac{i\left(\tilde{\eta}_{1}+\left(R^{k} \xi\right)_{1}\right)}{2}} \overline{e^{i\left(\eta_{1}+\left(R^{k-1} \xi\right)_{1}\right)}}\left|\widehat{\psi}_{a a}\left(\tilde{\eta}+R^{k} \xi\right) \widehat{\psi}_{a}\left(2 \eta+2 R^{k-1} \xi\right)\right| .
$$

Since $\eta_{1}=\pi=-\tilde{\eta}_{1}$, we have however $e^{\frac{i \tilde{\eta}_{1}}{2}}=-e^{\frac{i \eta_{1}}{2}}$ and $e^{i \tilde{\eta}_{1}}=e^{i \eta_{1}}$. Thus,

$$
\widehat{\psi}_{a}\left(\eta+R^{k} \xi\right) \overline{\widehat{\psi}_{a a}\left(2 \tilde{\eta}+2 R^{k+1} \xi\right)}=e^{\frac{i\left(\eta_{1}+\left(R^{k} \xi\right)_{1}\right)}{2}} \overline{e^{i\left(\eta_{1}+\left(R^{k+1} \xi\right)_{1}\right)}}\left|\widehat{\psi}_{a}\left(\eta+R^{k} \xi\right) \widehat{\psi}_{a a}\left(2 \tilde{\eta}+2 R^{k+1} \xi\right)\right|
$$

while

$$
\widehat{\psi}_{a a}\left(\tilde{\eta}+R^{k} \xi\right) \overline{\widehat{\psi}_{a}\left(2 \eta+2 R^{k-1} \xi\right)}=-e^{\frac{i\left(\eta_{1}+\left(R^{k} \xi\right)_{1}\right)}{2}} \overline{e^{i\left(\eta_{1}+\left(R^{k-1} \xi\right)_{1}\right)}}\left|\widehat{\psi}_{a a}\left(\tilde{\eta}+R^{k} \xi\right) \widehat{\psi}_{a}\left(2 \eta+2 R^{k-1} \xi\right)\right| .
$$

We obtain

$$
I_{5, j}=2 \int_{\mathbb{R}^{2}} \sum_{k \in \frac{Z}{N Z}} a_{k}(\xi)\left(\alpha_{k}(\xi) \overline{b_{k+1}(\xi)}-\beta_{k}(\xi) \overline{b_{k-1}(\xi)}\right) d \xi
$$

where

$$
\begin{aligned}
a_{k}(\xi) & =m_{j, k}\left(\eta+R^{k} \xi\right) e^{\frac{i\left(\eta_{1}+\left(R^{k} \xi\right)_{1}\right)}{2}}, \\
b_{k}(\xi) & =2 m_{j+1, k}\left(2 \eta+2 R^{k} \xi\right) e^{i\left(\eta_{1}+\left(R^{k} \xi\right)_{1}\right)}, \\
\alpha_{k}(\xi) & =\left|\widehat{\psi}_{a}\left(\eta+R^{k} \xi\right) \widehat{\psi}_{a a}\left(2 \tilde{\eta}+2 R^{k+1} \xi\right)\right|, \\
\beta_{k}(\xi) & =\left|\widehat{\psi}_{a a}\left(\tilde{\eta}+R^{k} \xi\right) \widehat{\psi}_{a}\left(2 \eta+2 R^{k-1} \xi\right)\right| .
\end{aligned}
$$

Hence, we can apply the following lemma.

Lemma 5 If $N$ is odd, then for all $a, b \in \mathbb{C}^{\frac{Z}{N Z}}$ and $\alpha, \beta \in \mathbb{R}_{+}^{\frac{Z}{N Z}}$, we have

$$
\begin{aligned}
2 R e \sum_{k \in \frac{Z}{N Z}} a_{k}\left(\alpha_{k} \overline{b_{k+1}}-\beta_{k} \overline{b_{k-1}}\right) \geq & 2\left(1-\cos \frac{\pi}{2 N}\right) \min _{k \in \frac{Z}{N Z}}\left(\min \left(\alpha_{k}, \beta_{k}\right)\right) \sum_{k \in \frac{Z}{N Z}}\left(\left|a_{k}\right|^{2}+\left|b_{k}\right|^{2}\right) \\
& -\sum_{k \in \in \frac{Z}{N Z}}\left(\left(\alpha_{k}+\beta_{k}\right)\left|a_{k}\right|^{2}+\left(\alpha_{k-1}+\beta_{k+1}\right)\left|b_{k}\right|^{2}\right) .
\end{aligned}
$$


Notice that, thanks to the symmetry properties of $\widehat{\psi}$ in $\mathbf{H 3}$, for all $\xi \in \mathbb{R}^{2},\left|\alpha_{k}\right|$ and $\left|\beta_{k}\right|$ have the same minimum value on $\left\{\omega \in \mathbb{R}^{2}:|\omega|=|\xi|\right\}$ and this value does not depends on $k$. We deduce that $\operatorname{Re}\left(I_{5, j}\right) \geq A_{j}-B_{j}$ where

$$
A_{j}=2\left(1-\cos \frac{\pi}{2 N}\right) \int_{\mathbb{R}^{2}\left\{\omega \in \mathbb{R}^{2}:|\omega|=|\xi|\right\}}\left|\alpha_{0}(\omega)\right| \sum_{k \in \frac{Z}{N Z}}\left(\left|a_{k}(\xi)\right|^{2}+\left|b_{k}(\xi)\right|^{2}\right) d \xi,
$$

and

$$
B_{j}=\int_{\mathbb{R}^{2}} \sum_{k \in \frac{Z}{N Z}}\left(\left(\alpha_{k}(\xi)+\beta_{k}(\xi)\right)\left|a_{k}(\xi)\right|^{2}+\left(\alpha_{k-1}(\xi)+\beta_{k+1}(\xi)\right)\left|b_{k}(\xi)\right|^{2}\right) d \xi
$$

Let us reverse the former changes of variables as follows

$$
\begin{aligned}
A_{j}= & 2\left(1-\cos \frac{\pi}{2 N}\right) \sum_{k \in \frac{Z}{N Z}}\left(\int_{\mathbb{R}^{2}} \min _{\left\{\omega \in \mathbb{R}^{2}:|\omega|=|\xi|\right\}}\left|\widehat{\psi}_{a}(\eta+\omega) \widehat{\psi}_{a a}(2 \tilde{\eta}+2 R \omega)\right|\left|m_{j, k}\left(\eta+R^{k} \xi\right)\right|^{2} d \xi\right. \\
& \left.+\int_{\mathbb{R}^{2}\left\{\omega \in \mathbb{R}^{2}:|\omega|=|\xi|\right\}}\left|\widehat{\psi}_{a}(\eta+\omega) \widehat{\psi}_{a a}(2 \tilde{\eta}+2 R \omega)\right|\left|2 m_{j+1, k}\left(2 \eta+2 R^{k} \xi\right)\right|^{2} d \xi\right) \\
= & 2\left(1-\cos \frac{\pi}{2 N}\right) \sum_{k \in \frac{Z}{N Z}}\left(\int_{\mathbb{R}^{2}\left\{\omega \in \mathbb{R}^{2}:|\omega-\eta|=|\xi-\eta|\right\}}\left|\widehat{\psi}_{a}(\omega) \widehat{\psi}_{a a}(2 R \omega)\right|\left|m_{j, k}(\xi)\right|^{2} d \xi\right. \\
& \left.+\int_{\mathbb{R}^{2}\left\{\omega \in \mathbb{R}^{2}:|\omega-2 \eta|=|\xi-2 \eta|\right\}}\left|\widehat{\psi}_{a}\left(2^{-1} \omega\right) \widehat{\psi}_{a a}(R \omega)\right|\left|m_{j+1, k}(\xi)\right|^{2} d \xi\right)
\end{aligned}
$$

We have therefore

$$
\sum_{j \in \mathbb{Z}} A_{j}=2\left(1-\cos \frac{\pi}{2 N}\right) \sum_{j \in \mathbb{Z}} \sum_{k \in \frac{\mathbb{Z}}{N \mathbb{Z}}} \int_{\mathbb{R}^{2}} F(\xi)\left|m_{j, k}(\xi)\right|^{2} d \xi
$$

where

$$
F(\xi)=\min _{\left\{\omega \in \mathbb{R}^{2}:|\omega-\eta|=|\xi-\eta|\right\}}\left|\widehat{\psi}_{a}(\omega) \widehat{\psi}_{a a}(2 R \omega)\right|+\min _{\left\{\omega \in \mathbb{R}^{2}:|\omega-2 \eta|=|\xi-2 \eta|\right\}}\left|\widehat{\psi}_{a}\left(2^{-1} \omega\right) \widehat{\psi}_{a a}(R \omega)\right| .
$$

On the other hand, applying the sames changes of variables, we have

$$
\begin{aligned}
B_{j}= & \sum_{k \in \frac{Z}{N Z}} \int_{\mathbb{R}^{2}}\left(\left(\left|\widehat{\psi}_{a}(\xi) \widehat{\psi}_{a a}(2 R \xi)\right|+\left|\widehat{\psi}_{a a}(\xi) \widehat{\psi}_{a}\left(2 R^{-1} \xi\right)\right|\right)\left|m_{j, k}(\xi)\right|^{2}\right) d \xi \\
& +\sum_{k \in \frac{Z}{N Z}} \int_{\mathbb{R}^{2}}\left(\left(\left|\widehat{\psi}_{a}\left(2^{-1} R^{-1} \xi\right) \widehat{\psi}_{a a}(\xi)\right|+\left|\widehat{\psi}_{a a}\left(2^{-1} R \xi\right) \widehat{\psi}_{a}(\xi)\right|\right)\left|m_{j+1, k}(\xi)\right|^{2}\right) d \xi .
\end{aligned}
$$

It follows that

$$
\sum_{j \in \mathbb{Z}} B_{j}=\sum_{j \in \mathbb{Z}} \sum_{k \in \frac{\mathbb{Z}}{\mathbb{N}}} \int_{\mathbb{R}^{2}} G(\xi)\left|m_{j, k}(\xi)\right|^{2} d \xi
$$

where

$$
G(\xi)=\sum_{j^{\prime}= \pm 1}\left(\left|\widehat{\psi}_{a}(\xi) \widehat{\psi}_{a a}\left(2^{j^{\prime}} R \xi\right)\right|+\left|\widehat{\psi}_{a a}(\xi) \widehat{\psi}_{a}\left(2^{j^{\prime}} R^{-1} \xi\right)\right|\right) .
$$

Joining all these together, we obtain

$$
\begin{aligned}
\sum_{j \in \mathbb{Z}} \operatorname{Re}\left(I_{5, j}\right) & \geq \sum_{j \in \mathbb{Z}}\left(A_{j}-B_{j}\right) \\
& =\int_{\mathbb{R}^{2}}\left(\sum_{j \in \mathbb{Z}} \sum_{k \in \frac{\mathbb{Z}}{N \mathbb{Z}}}\left|m_{j, k}(\xi)\right|^{2}\right)\left(2\left(1-\cos \frac{\pi}{2 N}\right) F(\xi)-G(\xi)\right) d \xi \\
& =\int_{\frac{\mathbb{R}^{2}}{\Gamma^{*}}}\left(\sum_{j \in \mathbb{Z}} \sum_{k \in \frac{\mathbb{Z}}{N \mathbb{Z}}}\left|m_{j, k}(\xi)\right|^{2}\right) \sum_{\gamma \in \Gamma^{*}}\left(2\left(1-\cos \frac{\pi}{2 N}\right) F(\xi+\gamma)-G(\xi+\gamma)\right) d \xi
\end{aligned}
$$


Finally, by exchanging $\widehat{\psi}_{a}$ and $\widehat{\psi}_{a a}$, we find a similar bound for $I_{6, j}$ and we deduce that

$$
I_{3}+I_{4} \geq \int_{\frac{\mathbb{R}^{2}}{\Gamma^{*}}}\left(\sum_{j \in \mathbb{Z}} \sum_{k \in \frac{\mathbb{Z}}{N \mathbb{Z}}}\left|m_{j, k}(\xi)\right|^{2}\right)\left(\Psi_{4}(\xi)-\Psi_{3}(\xi)\right) d \xi .
$$

\subsubsection{Proof of Lemma 4-(i)}

We have

$$
\begin{aligned}
J_{\mu}+J_{2 \mu}= & \sum_{j \in \mathbb{Z}} \sum_{k \in \frac{\mathbb{Z}}{N \mathbb{Z}}} \int_{\mathbb{R}^{2}} \hat{f}(\xi) \overline{\hat{\psi}\left(2^{-j} R^{-k} \xi\right) \hat{f}\left(\xi-2^{j} R^{k} \mu\right)} \widehat{\psi}\left(2^{-j} R^{-k} \xi-\mu\right) d \xi \\
& +\sum_{j \in \mathbb{Z}} \sum_{k \in \frac{\mathbb{Z}}{N \mathbb{Z}}} \int_{\mathbb{R}^{2}} \hat{f}(\xi) \overline{\widehat{\psi}\left(2^{-j} R^{-k} \xi\right) \hat{f}\left(\xi-2^{j+1} R^{k} \mu\right)} \widehat{\psi}\left(2^{-j} R^{-k} \xi-2 \mu\right) d \xi
\end{aligned}
$$

After the substitution of indices $j \mapsto j-1$, the second term on the right hand side reads

$$
\sum_{j \in \mathbb{Z}} \sum_{k \in \frac{\mathbb{Z}}{N \mathbb{Z}}} \int_{\mathbb{R}^{2}} \hat{f}(\xi) \overline{\hat{\psi}\left(2^{-j+1} R^{-k} \xi\right) \hat{f}\left(\xi-2^{j} R^{k} \mu\right)} \widehat{\psi}\left(2^{-j+1} R^{-k} \xi-2 \mu\right) d \xi
$$

Hence,

$$
J_{\mu}+J_{2 \mu}=\sum_{j \in \mathbb{Z}} \sum_{k \in \frac{\mathbb{Z}}{N \mathbb{Z}}} \int_{\mathbb{R}^{2}} \hat{f}(\xi) \overline{\hat{f}\left(\xi-2^{j} R^{k} \mu\right)} g_{j, k, \mu}(\xi) d \xi
$$

where

$$
g_{j, k, \mu}(\xi)=\overline{\widehat{\psi}\left(2^{-j} R^{-k} \xi\right)} \widehat{\psi}\left(2^{-j} R^{-k} \xi-\mu\right)+\overline{\widehat{\psi}\left(2^{-j+1} R^{-k} \xi\right)} \widehat{\psi}\left(2^{-j+1} R^{-k} \xi-2 \mu\right) .
$$

Observe that, by $\mathbf{H 2}$, we have

$$
\overline{\widehat{\psi}\left(2^{-j} R^{-k} \xi\right)} \widehat{\psi}\left(2^{-j} R^{-k} \xi-\mu\right)=e^{\frac{i \mu_{1}}{2}}\left|\widehat{\psi}\left(2^{-j} R^{-k} \xi\right) \widehat{\psi}\left(2^{-j} R^{-k} \xi-\mu\right)\right|,
$$

where $e^{\frac{i \mu_{1}}{2}}=e^{i \pi}=-1$. On the other hand, we have

$$
\overline{\widehat{\psi}\left(2^{-j+1} R^{-k} \xi\right)} \widehat{\psi}\left(2^{-j+1} R^{-k} \xi-2 \mu\right)=e^{i \mu_{1}}\left|\widehat{\psi}\left(2^{-j+1} R^{-k} \xi\right) \widehat{\psi}\left(2^{-j+1} R^{-k} \xi-2 \mu\right)\right|,
$$

where $e^{i \mu_{1}}=e^{i 2 \pi}=1$. Therefore,

$$
g_{j, k, \mu}(\xi)=-\left|\widehat{\psi}\left(2^{-j} R^{-k} \xi\right) \widehat{\psi}\left(2^{-j} R^{-k} \xi-\mu\right)\right|+\left|\widehat{\psi}\left(2^{-j+1} R^{-k} \xi\right) \widehat{\psi}\left(2^{-j+1} R^{-k} \xi-2 \mu\right)\right| .
$$

Going back to (22) and using Young inequality, we show that

$$
\begin{aligned}
2\left|J_{\mu}+J_{2 \mu}\right| & \leq 2 \sum_{j \in \mathbb{Z}} \sum_{k \in \frac{\mathbb{Z}}{N \mathbb{Z}}} \int_{\mathbb{R}^{2}}\left|\hat{f}(\xi) \hat{f}\left(\xi-2^{j} R^{k} \mu\right) g_{j, k, \mu}(\xi)\right| d \xi \\
& \leq \sum_{j \in \mathbb{Z}} \sum_{k \in \frac{\mathbb{Z}}{N \mathbb{Z}}} \int_{\mathbb{R}^{2}}\left(|\hat{f}(\xi)|^{2}+\left|\hat{f}\left(\xi-2^{j} R^{k} \mu\right)\right|^{2}\right)\left|g_{j, k, \mu}(\xi)\right| d \xi
\end{aligned}
$$

where

$$
\begin{aligned}
\int_{\mathbb{R}^{2}}\left(|\hat{f}(\xi)|^{2}+\left|\hat{f}\left(\xi-2^{j} R^{k} \mu\right)\right|^{2}\right)\left|g_{j, k, \mu}(\xi)\right| d \xi & =\int_{\mathbb{R}^{2}}|\hat{f}(\xi)|^{2}\left(\left|g_{j, k, \mu}(\xi)\right|+\left|g_{j, k, \mu}\left(\xi+2^{j} R^{k} \mu\right)\right|\right) d \xi \\
& =\int_{\mathbb{R}^{2}}|\hat{f}(\xi)|^{2}\left(\left|g_{j, k, \mu}(\xi)\right|+\left|g_{j, k,-\mu}(\xi)\right|\right) d \xi
\end{aligned}
$$

Thus, we have

$$
2\left|J_{\mu}+J_{2 \mu}\right| \leq \int_{\mathbb{R}^{2}}|\hat{f}(\xi)|^{2} \sum_{j \in \mathbb{Z}} \sum_{k \in \frac{\mathbb{Z}}{N \mathbb{Z}}}\left(\left|g_{j, k, \mu}(\xi)\right|+\left|g_{j, k,-\mu}(\xi)\right|\right) d \xi=\int_{\mathbb{R}^{2}}|\hat{f}(\xi)|^{2} \Phi_{1}(\xi) d \xi .
$$




\subsubsection{Proof of Lemma 4-(ii)}

The proof is similar to that of Lemma 4-(i) where we change the dilation by 2 into the rotation $R$. We have

$$
\begin{aligned}
J_{3 \eta}+J_{3 \tilde{\eta}}= & \sum_{j \in \mathbb{Z}} \sum_{k \in \mathbb{Z}} \int_{\mathbb{R}^{2}} \hat{f}(\xi) \overline{\widehat{\psi}\left(2^{-j} R^{-k} \xi\right) \hat{f}\left(\xi-2^{j} R^{k}(3 \eta)\right)} \widehat{\psi}\left(2^{-j} R^{-k} \xi-3 \eta\right) d \xi \\
& +\sum_{j \in \mathbb{Z}} \sum_{k \in \frac{\mathbb{Z}}{N \mathbb{Z}}} \int_{\mathbb{R}^{2}} \hat{f}(\xi) \overline{\widehat{\psi}\left(2^{-j} R^{-k} \xi\right) \hat{f}\left(\xi-2^{j} R^{k+1}(3 \eta)\right)} \widehat{\psi}\left(2^{-j} R^{-k} \xi-3 \tilde{\eta}\right) d \xi
\end{aligned}
$$

After a change of indices, the second term on the right hand side reads

$$
\sum_{j \in \mathbb{Z}} \sum_{k \in \frac{Z}{N Z}} \int_{\mathbb{R}^{2}} \hat{f}(\xi) \overline{\hat{\psi}\left(2^{-j} R^{-k+1} \xi\right) \hat{f}\left(\xi-2^{j} R^{k}(3 \eta)\right)} \widehat{\psi}\left(2^{-j} R^{-k+1} \xi-3 \tilde{\eta}\right) d \xi .
$$

Hence,

$$
J_{3 \eta}+J_{3 \tilde{\eta}}=\sum_{j \in \mathbb{Z}} \sum_{k \in \frac{\mathbb{Z}}{\mathrm{NZ}}} \int_{\mathbb{R}^{2}} \hat{f}(\xi) \overline{\hat{f}\left(\xi-2^{j} R^{k}(3 \eta)\right)} h_{j, k, 3 \eta}(\xi) d \xi
$$

where

$$
h_{j, k, 3 \eta}(\xi)=\overline{\widehat{\psi}\left(2^{-j} R^{-k} \xi\right)} \widehat{\psi}\left(2^{-j} R^{-k} \xi-3 \eta\right)+\overline{\left.\widehat{\psi}\left(2^{-j} R^{-k+1} \xi\right)\right)} \widehat{\psi}\left(2^{-j} R^{-k+1} \xi-3 \tilde{\eta}\right)
$$

Notice that, by $\mathbf{H 2}$, we have

$$
\overline{\widehat{\psi}\left(2^{-j} R^{-k} \xi\right)} \widehat{\psi}\left(2^{-j} R^{-k} \xi-3 \eta\right)=e^{\frac{i 3 \eta_{1}}{2}}\left|\widehat{\psi}\left(2^{-j} R^{-k} \xi\right) \widehat{\psi}\left(2^{-j} R^{-k} \xi-3 \eta\right)\right|
$$

where $e^{\frac{i 3 \eta_{1}}{2}}=e^{\frac{i 3 \pi}{2}}=-i$. On the other hand, we have

$$
\overline{\widehat{\psi}\left(2^{-j} R^{-k+1} \xi\right)} \widehat{\psi}\left(2^{-j} R^{-k+1} \xi-3 \tilde{\eta}\right)=e^{\frac{i 3(\tilde{\eta})_{1}}{2}}\left|\widehat{\psi}\left(2^{-j} R^{-k+1} \xi\right) \widehat{\psi}\left(2^{-j} R^{-k+1} \xi-3 \tilde{\eta}\right)\right|
$$

where $e^{\frac{-i 3 \tilde{1}_{1}}{2}}=e^{-\frac{i 3 \pi}{2}}=i$. Therefore,

$$
h_{j, k, 3 \eta}(\xi)=-i\left|\widehat{\psi}\left(2^{-j} R^{-k} \xi\right) \widehat{\psi}\left(2^{-j} R^{-k} \xi-3 \eta\right)\right|+i\left|\widehat{\psi}\left(2^{-j} R^{-k+1} \xi\right) \widehat{\psi}\left(2^{-j} R^{-k+1} \xi-3 \tilde{\eta}\right)\right| .
$$

Going back to (23) and using Young inequality, we show that

$$
\begin{aligned}
2\left|J_{3 \eta}+J_{-3 \eta}\right| & \leq 2 \sum_{j \in \mathbb{Z}} \sum_{k \in \frac{\mathbb{Z}}{N \mathbb{Z}}} \int_{\mathbb{R}^{2}}\left|\hat{f}(\xi) \hat{f}\left(\xi-2^{j} R^{k}(3 \eta)\right) h_{j, k, 3 \eta}(\xi)\right| d \xi \\
& \leq \sum_{j \in \mathbb{Z}} \sum_{k \in \frac{\mathbb{Z}}{N \mathbb{Z}}} \int_{\mathbb{R}^{2}}\left(|\hat{f}(\xi)|^{2}+\left|\hat{f}\left(\xi-2^{j} R^{k}(3 \eta)\right)\right|^{2}\right)\left|h_{j, k, 3 \eta}(\xi)\right| d \xi
\end{aligned}
$$

where

$$
\begin{aligned}
\int_{\mathbb{R}^{2}}\left(|\hat{f}(\xi)|^{2}+\left|\hat{f}\left(\xi-2^{j} R^{k}(3 \eta)\right)\right|^{2}\right)\left|h_{j, k, 3 \eta}(\xi)\right| d \xi & =\int_{\mathbb{R}^{2}}|\hat{f}(\xi)|^{2}\left(\left|h_{j, k, 3 \eta}(\xi)\right|+\left|h_{j, k, 3 \eta}\left(\xi+2^{j} R^{k}(3 \eta)\right)\right|\right) d \xi \\
& =\int_{\mathbb{R}^{2}}|\hat{f}(\xi)|^{2}\left(\left|h_{j, k, 3 \eta}(\xi)\right|+\left|h_{j, k,-3 \eta}(\xi)\right|\right) d \xi
\end{aligned}
$$

Thus, we have

$$
2\left|J_{3 \eta}+J_{3 \tilde{\eta}}\right| \leq \int_{\mathbb{R}^{2}}|\hat{f}(\xi)|^{2} \Phi_{2}(\xi) d \xi
$$




\subsubsection{Proof of Lemma 4-(iii)}

We have

$$
\begin{aligned}
J_{\nu}+J_{\tilde{\nu}}= & \sum_{j \in \mathbb{Z}}\left(\sum_{k \in \frac{Z}{N \mathbb{Z}}} \int_{\mathbb{R}^{2}} \hat{f}(\xi) \overline{\widehat{\psi}\left(2^{-j} R^{k} \xi\right) \hat{f}\left(\xi-2^{j} R^{-k} \nu\right)} \widehat{\psi}\left(2^{-j} R^{k} \xi-\nu\right) d \xi\right. \\
& \left.+\sum_{k \in \frac{Z}{N \mathbb{Z}}} \int_{\mathbb{R}^{2}} \hat{f}(\xi) \overline{\widehat{\psi}\left(2^{-j} R^{k} \xi\right) \hat{f}\left(\xi-2^{j} R^{-k} \tilde{\nu}\right)} \widehat{\psi}\left(2^{-j} R^{k} \xi-\tilde{\nu}\right) d \xi\right) .
\end{aligned}
$$

By developing $\widehat{\psi}$ as $\widehat{\psi}_{a}+\widehat{\psi}_{a a}$, we show that $J_{\nu}+J_{\tilde{\nu}}=\sum_{j \in \mathbb{Z}}\left(J_{1, j}+J_{2, j}\right)$, where

$$
\begin{aligned}
J_{1, j}= & \sum_{k \in \in \frac{Z}{N Z}} \int_{\mathbb{R}^{2}} \hat{f}(\xi) \overline{\widehat{\psi}_{a}\left(2^{-j} R^{-k} \xi\right) \hat{f}\left(\xi-2^{j} R^{k} \nu\right)} \widehat{\psi}_{a}\left(2^{-j} R^{-k} \xi-\nu\right) d \xi \\
& +\sum_{k \in \frac{Z}{N Z}} \int_{\mathbb{R}^{2}} \hat{f}(\xi) \overline{\widehat{\psi}_{a a}\left(2^{-j} R^{-k} \xi\right) \hat{f}\left(\xi-2^{j} R^{k} \tilde{\nu}\right)} \widehat{\psi}_{a a}\left(2^{-j} R^{-k} \xi-\tilde{\nu}\right) d \xi
\end{aligned}
$$

and

$$
\begin{aligned}
J_{2, j}= & \sum_{k \in \frac{Z}{N Z}} \int_{\mathbb{R}^{2}} \hat{f}(\xi) \overline{\hat{\psi}_{a a}\left(2^{-j} R^{-k} \xi\right) \hat{f}\left(\xi-2^{j} R^{k} \nu\right)} \widehat{\psi}_{a a}\left(2^{-j} R^{-k} \xi-\nu\right) d \xi \\
& +\sum_{k \in \frac{Z}{N Z}} \int_{\mathbb{R}^{2}} \hat{f}(\xi) \overline{\widehat{\psi}_{a}\left(2^{-j} R^{-k} \xi\right) \hat{f}\left(\xi-2^{j} R^{k} \tilde{\nu}\right)} \widehat{\psi}_{a}\left(2^{-j} R^{-k} \xi-\tilde{\nu}\right) d \xi .
\end{aligned}
$$

Let us focus on $J_{1, j}$. Since $e^{-\frac{\nu_{1}}{2}}=e^{-i \frac{\pi}{2}}=-i$ and $e^{-\frac{\nu_{1}}{2}}=e^{i \frac{\pi}{2}}=i$, we have, by $\mathbf{H 2}$,

$$
\overline{\widehat{\psi}_{a}\left(2^{-j} R^{-k} \xi\right)} \widehat{\psi}_{a}\left(2^{-j} R^{-k} \xi-\nu\right)=-i\left|\widehat{\psi}_{a}\left(2^{-j} R^{-k} \xi\right) \widehat{\psi}_{a}\left(2^{-j} R^{-k} \xi-\nu\right)\right|
$$

and

$$
\overline{\widehat{\psi}_{a a}\left(2^{-j} R^{-k} \xi\right)} \widehat{\psi}_{a a}\left(2^{-j} R^{-k} \xi-\tilde{\nu}\right)=i\left|\widehat{\psi}_{a a}\left(2^{-j} R^{-k} \xi\right) \widehat{\psi}_{a a}\left(2^{-j} R^{-k} \xi-\tilde{\nu}\right)\right| .
$$

It follows that

$$
\begin{aligned}
J_{1, j}= & -i \sum_{k \in \frac{Z}{N Z}} \int_{\mathbb{R}^{2}} \hat{f}(\xi)\left|\widehat{\psi}_{a}\left(2^{-j} R^{-k} \xi\right) \widehat{\psi}_{a}\left(2^{-j} R^{-k} \xi-\nu\right)\right| \overline{\hat{f}\left(\xi-2^{j} R^{k} \nu\right)} d \xi \\
& +i \sum_{k \in \frac{Z}{N Z}} \int_{\mathbb{R}^{2}} i \hat{f}(\xi)\left|\widehat{\psi}_{a a}\left(2^{-j} R^{-k} \xi\right) \widehat{\psi}_{a a}\left(2^{-j} R^{-k} \xi-\tilde{\nu}\right)\right| \overline{\hat{f}\left(\xi-2^{j} R^{k} \tilde{\nu}\right)} d \xi
\end{aligned}
$$

In the first integrals, we make the changes of variables $\xi \mapsto 2^{j+1} R^{k} \eta+\xi$, while in the other integrals, we apply $\xi \mapsto 2^{j+1} R^{k+1} \eta+\xi$. Since $2 \eta-\nu=-\tilde{\eta}$ and $2 R \eta-\tilde{\nu}=-\eta$, we obtain

$$
\begin{aligned}
J_{1, j}= & -i \sum_{k \in \in} \int_{\mathbb{R}^{2}} \hat{f}\left(2^{j+1} R^{k} \eta+\xi\right)\left|\widehat{\psi}_{a}\left(2 \eta+2^{-j} R^{-k} \xi\right) \widehat{\psi}_{a}\left(-\tilde{\eta}+2^{-j} R^{-k} \xi\right)\right| \overline{\hat{f}\left(-2^{j} R^{k+1} \eta+\xi\right)} d \xi \\
& +i \sum_{k \in \frac{Z}{N Z}} \int_{\mathbb{R}^{2}} \hat{f}\left(2^{j+1} R^{k+1} \eta+\xi\right)\left|\widehat{\psi}_{a a}\left(2 \tilde{\eta}+2^{-j} R^{-k} \xi\right) \widehat{\psi}_{a a}\left(-\eta+2^{-j} R^{-k} \xi\right)\right| \overline{\hat{f}\left(-2^{j} R^{k} \eta+\xi\right)} d \xi \\
= & \sum_{k \in \frac{Z}{N Z}} \int_{\mathbb{R}^{2}} \hat{f}\left(2^{j+1} R^{k} \eta+\xi\right)\left(\left|\widehat{\psi}_{a}\left(2 \eta+2^{-j} R^{-k} \xi\right) \widehat{\psi}_{a}\left(-\tilde{\eta}+2^{-j} R^{-k} \xi\right)\right| \overline{i \hat{f}\left(-2^{j} R^{k+1} \eta+\xi\right)}\right. \\
= & \left.\quad-\left|\widehat{\psi}_{a a}\left(2 \tilde{\eta}+2^{-j} R^{-k+1} \xi\right) \widehat{\psi}_{a a}\left(-\eta+2^{-j} R^{-k+1} \xi\right)\right| \overline{i \hat{f}\left(-2^{j} R^{k-1} \eta+\xi\right)}\right) d \xi \\
& \int_{k \in \frac{Z}{N Z}} a_{\mathbb{R}^{2}}(\xi)\left(\alpha_{k}(\xi) \overline{b_{k+1}(\xi)}-\beta_{k}(\xi) \overline{b_{k-1}(\xi)}\right) d \xi,
\end{aligned}
$$


where

$$
\begin{aligned}
a_{k}(\xi) & =\hat{f}\left(2^{j+1} R^{k} \eta+\xi\right), \\
b_{k}(\xi) & =i \hat{f}\left(-2^{j} R^{k} \eta+\xi\right), \\
\alpha_{k}(\xi) & =\left|\widehat{\psi}_{a}\left(2 \eta+2^{-j} R^{-k} \xi\right) \widehat{\psi}_{a}\left(-\tilde{\eta}+2^{-j} R^{-k} \xi\right)\right|, \\
\beta_{k}(\xi) & =\left|\widehat{\psi}_{a a}\left(2 \tilde{\eta}+2^{-j} R^{-k+1} \xi\right) \widehat{\psi}_{a a}\left(-\eta+2^{-j} R^{-k+1} \xi\right)\right| .
\end{aligned}
$$

Thus, we can apply once again Lemma 5 to show that $2 \operatorname{Re}\left(J_{1, j}\right) \geq A_{j}-B_{j}$, where

$$
A_{j}=2\left(1-\cos \frac{\pi}{2 N}\right) \int_{\mathbb{R}^{2}\left\{\omega \in \mathbb{R}^{2}:|\omega|=|\xi|\right\}}\left|\alpha_{0}(\omega)\right| \sum_{k \in \frac{\mathbb{Z}}{N \mathbb{Z}}}\left(\left|a_{k}(\xi)\right|^{2}+\left|b_{k}(\xi)\right|^{2}\right) d \xi,
$$

and

$$
B_{j}=\int_{\mathbb{R}^{2}} \sum_{k \in \frac{\mathbb{Z}}{N \mathbb{Z}}}\left(\left(\alpha_{k}(\xi)+\beta_{k}(\xi)\right)\left|a_{k}(\xi)\right|^{2}+\left(\alpha_{k-1}(\xi)+\beta_{k+1}(\xi)\right)\left|b_{k}(\xi)\right|^{2}\right) d \xi .
$$

Let us reverse the former change of variables. We have

$$
\begin{aligned}
A_{j}= & 2\left(1-\cos \frac{\pi}{2 N}\right) \sum_{k \in \frac{Z}{N Z}}\left(\int_{\mathbb{R}^{2}} \min _{\left\{\omega \in \mathbb{R}^{2}:|\omega|=|\xi|\right\}}\left|\widehat{\psi}_{a}\left(2 \eta+2^{-j} \omega\right) \widehat{\psi}_{a}\left(-\tilde{\eta}+2^{-j} \omega\right)\right|\left|\hat{f}\left(2^{j+1} R^{k} \eta+\xi\right)\right|^{2} d \xi\right. \\
& \left.+\int_{\mathbb{R}^{2}} \min _{\left\{\omega \in \mathbb{R}^{2}:|\omega|=|\xi|\right\}}\left|\widehat{\psi}_{a}\left(2 \eta+2^{-j} \omega\right) \widehat{\psi}_{a}\left(-\tilde{\eta}+2^{-j} \omega\right)\right|\left|\hat{f}\left(-2^{j} R^{k} \eta+\xi\right)\right|^{2} d \xi\right) \\
= & 2\left(1-\cos \frac{\pi}{2 N}\right) \int_{\mathbb{R}^{2}}\left(\sum_{k \in \frac{Z}{N Z}} \sum_{\gamma \in\{2 \eta,-\tilde{\eta}\}} \min _{\left\{\omega \in \mathbb{R}^{2}:|\omega|=\left|2^{-j} \xi-R^{k} \gamma\right|\right\}}\left|\widehat{\psi}_{a}(2 \eta+\omega) \widehat{\psi}_{a}(-\tilde{\eta}+\omega)\right|\right)|\hat{f}(\xi)|^{2} d \xi
\end{aligned}
$$

We infer that

$$
\sum_{j \in \mathbb{Z}} A_{j}=2\left(1-\cos \frac{\pi}{2 N}\right) \int_{\mathbb{R}^{2}} H(\xi)|\hat{f}(\xi)|^{2} d \xi
$$

where

$$
H(\xi)=\sum_{j \in \mathbb{Z}} \sum_{k \in \frac{Z}{N \mathbb{Z}}} \sum_{\gamma \in\{2 \eta,-\tilde{\eta}\}} \min _{\left\{\omega \in \mathbb{R}^{2}:|\omega|=\left|2^{-j} \xi-R^{k} \gamma\right|\right\}}\left|\widehat{\psi}_{a}(2 \eta+\omega) \widehat{\psi}_{a}(-\tilde{\eta}+\omega)\right| .
$$

Applying the sames changes of variables in $B_{j}$, we get

$$
\begin{aligned}
B_{j}= & \sum_{k \in \frac{Z}{N Z}} \int_{\mathbb{R}^{2}}|\hat{f}(\xi)|^{2}\left(\left|\widehat{\psi}_{a}\left(2^{-j} R^{-k} \xi\right) \widehat{\psi}_{a}\left(2^{-j} R^{-k} \xi-\nu\right)\right|+\left|\widehat{\psi}_{a a}\left(2^{-j} R^{-k+1} \xi\right) \widehat{\psi}_{a a}\left(2^{-j} R^{-k+1} \xi-\tilde{\nu}\right)\right|\right) d \xi \\
& +\sum_{k \in \frac{Z}{N Z}} \int_{\mathbb{R}^{2}}|\hat{f}(\xi)|^{2}\left(\left|\widehat{\psi}_{a}\left(2^{-j} R^{-k+1} \xi\right) \widehat{\psi}_{a}\left(2^{-j} R^{-k+1} \xi+\nu\right)\right|+\left|\widehat{\psi}_{a a}\left(2^{-j} R^{-k} \xi\right) \widehat{\psi}_{a a}\left(2^{-j} R^{-k} \xi+\tilde{\nu}\right)\right|\right) d \xi \\
= & \int_{\mathbb{R}^{2}}|\hat{f}(\xi)|^{2} \sum_{\gamma \in\{\nu,-\nu\}} \sum_{k \in \frac{Z}{N Z}}\left(\left|\widehat{\psi}_{a}\left(2^{-j} R^{-k} \xi\right) \widehat{\psi}_{a}\left(2^{-j} R^{-k} \xi-\gamma\right)\right|+\left|\widehat{\psi}_{a a}\left(2^{-j} R^{-k} \xi\right) \widehat{\psi}_{a a}\left(2^{-j} R^{-k} \xi-\tilde{\gamma}\right)\right|\right) d \xi .
\end{aligned}
$$

Joining all these together, we obtain

$$
\begin{aligned}
2 \sum_{j \in \mathbb{Z}} \operatorname{Re}\left(J_{1, j}\right) & \geq \sum_{j \in \mathbb{Z}}\left(A_{j}-B_{j}\right) \\
& =\int_{\mathbb{R}^{2}}|\hat{f}(\xi)|^{2}\left(2\left(1-\cos \frac{\pi}{2 N}\right) H(\xi)\right. \\
& \left.-\sum_{\gamma \in\{\nu,-\nu\}} \sum_{j \in \mathbb{Z}} \sum_{k \in \frac{\mathbb{Z}}{N \mathbb{Z}}}\left(\left|\widehat{\psi}_{a}\left(2^{-j} R^{-k} \xi\right) \widehat{\psi}_{a}\left(2^{-j} R^{-k} \xi-\gamma\right)\right|+\left|\widehat{\psi}_{a a}\left(2^{-j} R^{-k} \xi\right) \widehat{\psi}_{a a}\left(2^{-j} R^{-k} \xi-\tilde{\gamma}\right)\right|\right)\right) d \xi .
\end{aligned}
$$


Finally, by exchanging $\widehat{\psi}_{a}$ and $\widehat{\psi}_{a a}$, we find a similar bound for $J_{2, j}$ and we deduce that

$$
2 \operatorname{Re}\left(J_{\nu}+J_{\tilde{\nu}}\right) \geq \int_{\mathbb{R}^{2}}|\hat{f}(\xi)|^{2}\left(\Phi_{4}(\xi)-\Phi_{3}(\xi)\right) d \xi
$$

\subsubsection{Proof of Lemma 5}

First, we show that if $N$ is odd, then we have, for all $a, b \in \mathbb{C} \frac{\mathbb{Z}}{N \mathbb{Z}}$,

$$
\sum_{k \in \frac{\mathbb{Z}}{N \mathbb{Z}}}\left(\left|a_{k}-b_{k-1}\right|^{2}+\left|a_{k}+b_{k+1}\right|^{2}\right) \geq 2\left(1-\cos \frac{\pi}{2 N}\right) \sum_{k \in \frac{\mathbb{Z}}{N \mathbb{Z}}}\left(\left|a_{k}\right|^{2}+\left|b_{k}\right|^{2}\right) .
$$

To prove this inequality, we need to change the notation as follows. As $N$ is odd, put $N=2 n+1$, and for all $k \in\{0, \cdots, 4 n+1\}$, set

$$
c_{k}= \begin{cases}a_{[k]}, & \text { if } k \text { is even, } \\ b_{[k]}, & \text { if } k \text { is odd, }\end{cases}
$$

where $[k]$ is the equivalence class of $k$ in $\frac{\mathbb{Z}}{N \mathbb{Z}}$. Inequality (24) becomes

$$
\sum_{k=0}^{4 n}\left|c_{k}+(-1)^{k} c_{k+1}\right|^{2}+\left|c_{4 n+1}-c_{0}\right|^{2} \geq 2\left(1-\cos \frac{\pi}{4 n+2}\right) \sum_{k=0}^{4 n+1}\left|c_{k}\right|^{2}, \quad \forall c \in \mathbb{C}^{4 n+2}
$$

It can be written in matrix form as $\|A c\|^{2} \geq 2\left(1-\cos \frac{\pi}{4 n+2}\right)\|c\|^{2}$, or equivalently as

$$
\|A c\| \geq\left|1-e^{-i \frac{\pi}{4 n+2}}\right|\|c\|
$$

where $\|\cdot\|$ is the Euclidean norm and

$$
A=\left(\begin{array}{ccccc}
1 & 1 & & & \\
& 1 & -1 & & \\
& & 1 & 1 & \\
& & & \ddots & \ddots \\
-1 & & & & 1
\end{array}\right)
$$

Since $A^{T} A=A A^{T}$, where $A^{T}$ denotes the transpose of $A$, the matrix $A$ is diagonalizable in an orthonormal basis. Let $\lambda$ be its eigenvalue with smallest modulus. Proving (26) amounts to show that $|\lambda|=\left|1-e^{-i \frac{\pi}{4 n+2}}\right|$. The characteristic polynomial of $A$ is $(1-\lambda)^{4 n+2}+1$ and we deduce that $\lambda=$ $1-i e^{2 i \frac{l \pi}{4 n+2}}$ for $l \in \frac{\mathbb{Z}}{(4 n+2) \mathbb{Z}}$. The modulus $|\lambda|$ is therefore minimal for $l \equiv 3 n+1$ and we have $|\lambda|=\left|1-i e^{2 i \frac{3 n+1}{4 n+2} \pi}\right|=\left|1-e^{-i \frac{\pi}{4 n+2}}\right|$. Hence, (26) and (24) are proved.

Next, using the binomial formula, we show that

$$
\begin{aligned}
2 \operatorname{Re} \sum_{k \in \frac{\mathbb{Z}}{N \mathbb{Z}}} a_{k}\left(\alpha_{k} \overline{b_{k+1}}-\beta_{k} \overline{b_{k-1}}\right)= & \sum_{k \in \frac{\mathbb{Z}}{N \mathbb{Z}}}\left(\alpha_{k}\left|a_{k}-b_{k+1}\right|^{2}+\beta_{k}\left|a_{k}+b_{k-1}\right|^{2}\right) \\
& -\sum_{k \in \frac{\mathbb{Z}}{N \mathbb{Z}}}\left(\left(\alpha_{k}+\beta_{k}\right)\left|a_{k}\right|^{2}+\left(\alpha_{k-1}+\beta_{k+1}\right)\left|b_{k}\right|^{2}\right),
\end{aligned}
$$

where, thanks to the above,

$$
\begin{aligned}
\sum_{k \in \frac{\mathbb{Z}}{N \mathbb{Z}}}\left(\alpha_{k}\left|a_{k}-b_{k+1}\right|^{2}+\beta_{k}\left|a_{k}+b_{k-1}\right|^{2}\right) & \geq \min _{k \in \frac{\mathbb{Z}}{N \mathbb{Z}}} \min \left(\alpha_{k}, \beta_{k}\right) \sum_{k \in \frac{\mathbb{Z}}{N \mathbb{Z}}}\left(\left|a_{k}-b_{k+1}\right|^{2}+\left|a_{k}+b_{k-1}\right|^{2}\right) \\
& \geq 2\left(1-\cos \frac{\pi}{2 N}\right) \min _{k \in \frac{\mathbb{Z}}{N \mathbb{Z}}} \min \left(\alpha_{k}, \beta_{k}\right) \sum_{k \in \frac{\mathbb{Z}}{N \mathbb{Z}}}\left(\left|a_{k}\right|^{2}+\left|b_{k}\right|^{2}\right)
\end{aligned}
$$




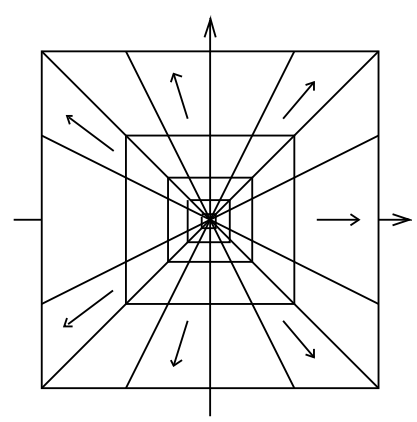

(a)

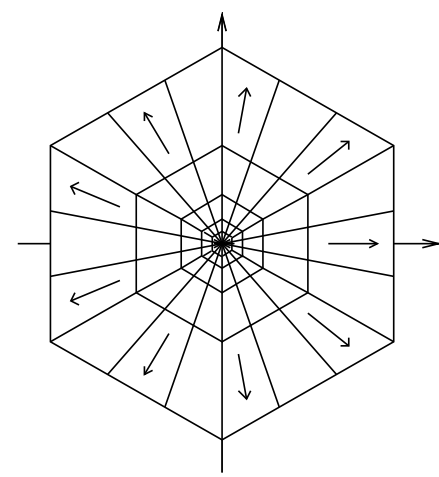

(b)

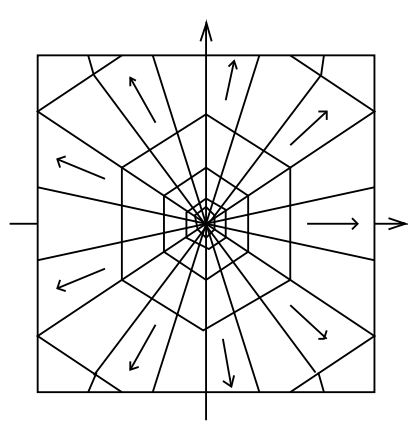

(c)

Figure 5: Three examples of potential implementations for squarely sampled images - (a) and (c) — and hexagonally sampled images-(b).

\section{$5 \quad$ Numerical implementation}

In order to demonstrate the potential of the proposed approach, we give a first numerical implementation. This task raises several core issues that are addressed below.

\subsection{Adaptation to a non-rotation invariant lattice}

The frequency tiling displayed in Figure 1-(b) is not adapted to digital images that are generally sampled on a square lattice and have therefore a square reciprocal cell. An easy solution is to replace some rotations by shear mappings as it is done for the discrete curvelet transform [3] and the shearlet transform [14].

In order not to get off the above theorems and unlike what is generally done for discrete directional bases, the wavelet $\psi \circ D$, for a given mapping $D$, has to be translated along the lattice $D^{-1} \Gamma$. As the transform in implemented in the Fourier domain, this lattice does not have necessary to be a sublattice of the lattice $\mathbb{Z}^{2}$ on which the image is sample. It has however to be a sublattice of the periodization lattice $N \mathbb{Z} \times M \mathbb{Z}$ for a $N \times M$-image. Tilings that are generally considered, in particular those of [12], are therefore not adapted.

Moreover, since the number of directions has to be odd, the basis cannot be invariant by rotation of angle $\pi / 2$. We propose therefore to use the frequency tiling of Figure 5-(a) that is well suited to $2^{n} \times 2^{m}$-images. In this example, only 6 of the 7 directional wavelets are obtained by compositions of rotations and shear mappings with a mother wavelet $\psi$. The seventh wavelet is designed separately. The latter and $\psi$ both satisfy equation (2) with the same $\delta$ and $\tau$, but for different angular resolutions - see Figure 6. One can prove that Proposition 1 can be extended to this case. For each scale $j$, we have therefore an orthonormal basis for $W_{j}=\bigoplus_{k \in\{1, \cdots, 7\}} W_{j, k}$.

Notice that one could obtain more uniform angular resolutions for the 3-loosely-horizontal directions with $\left(3 \cdot 2^{n}\right) \times 2^{m}$-images. In order to have wavelets with nearly the same angular resolution in each direction, it would be however more suitable to consider images that are sampled on an hexagonal lattice - see Figure 5-(b). Recall, by the way, that hexagonal lattices have several notable properties such as requiring the least number of samples to represent images whose spectrum is supported on a disc. On the other hand, since most of images that are produced nowadays are sampled on a square lattice, it might be appropriate to adapt this hexagonal transform to squarely sampled images by shifting some 


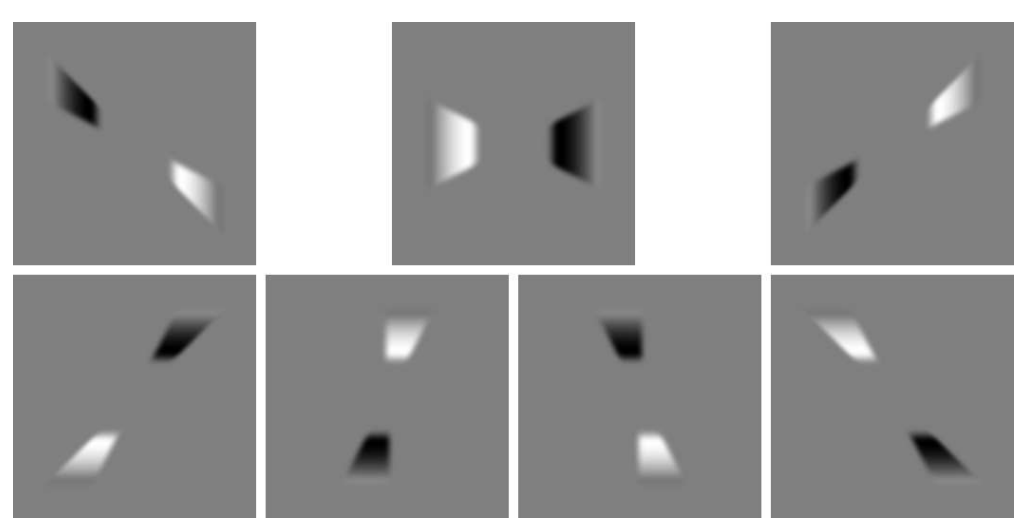

Figure 6: Fourier transforms of the primal wavelets. Only imaginary parts are shown.

of their Fourier coefficients as it was done in [13] - See Figure 5-(c). This third solution would be well suited as the transform, based on bandlimited wavelets, has to be implemented in the Fourier domain.

\subsection{Processing of the finest and coarsest scales}

Since the frequency support of wavelets at the finest scale exceed the cutoff frequency, their processing is a genuine issue, especially when our aim is to avoid aliasing. The Fourier transforms are not periodized as it is done, for instance, in [3]. They are roughly cut as if the image was upsampled by zero padding. As a consequence, fine scale wavelets have low decay. A periodization would however have the same effect since wavelets are not translated on a sublattice of $\mathbb{Z}^{2}$ or, in other words, Fourier transforms of wavelets cannot be periodized along $\left(\mathbb{Z}^{2}\right)^{*}$.

Coarse scales can be processed with separable Meyer scaling function as in [3]. It causes however a break in the connections between scales and generates therefore a low frequency residue on dual wavelets. To avoid this issue, wavelets are applied down to the coarsest scale with some approximations in accordance with the sampling theory.

\subsection{Experimental results}

The primal wavelet transform is implemented in the Fourier domain. Since wavelets are not translated on square lattices, we used FFT for non-square lattices as in [13]. Up to now, we do not know how to obtain an explicit expression of the dual basis. It is therefore computed by conjugate gradient. Figure 7 shows the 7 primal wavelets and the computed dual wavelets. One can observe that the two families are rather close to each other. This is not surprising since primal wavelets at a same scale form an orthonormal system. However the orthogonality does not stand up to dilation. As a consequence, one can notice a light high frequency residue on dual wavelets. We do not know yet whether these artifacts are due to the adopted processing of the finest and coarsest scales whose effects reverberate through scales. In figure 8 , one of the primal wavelets, at the finest scale, and the associated dual wavelet are displayed. As it is mentioned above, they have low decay.

We obtained better results when the analysis is done with the primal wavelet. In such a case, the direct transform is fast, while the inverse transform is built on an iterative process. It is done by conjugate gradient in the Fourier domain, hence avoiding a recurrent use of the Fourier transform. 


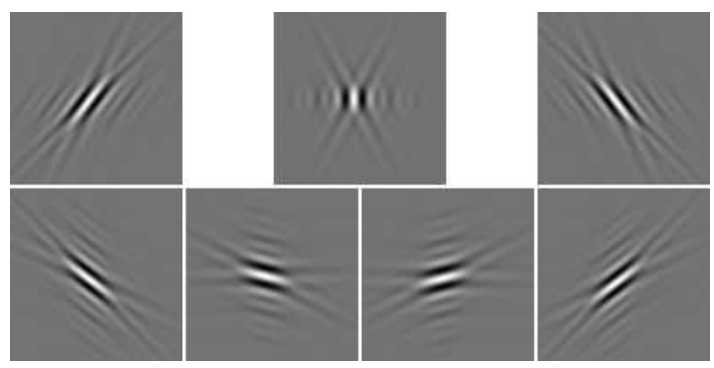

(a)

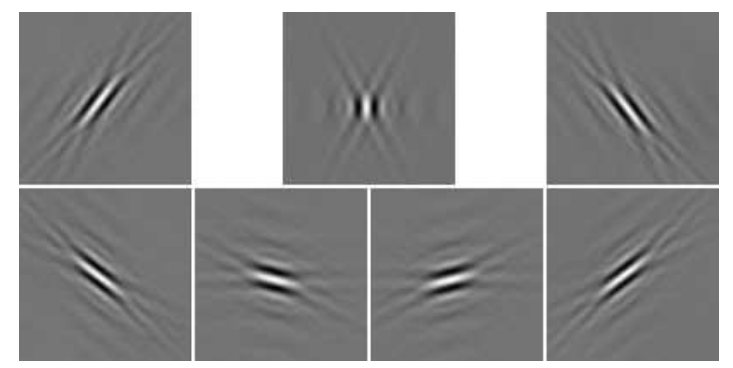

(b)

Figure 7: (a) Primal wavelets. (b) Dual wavelets.

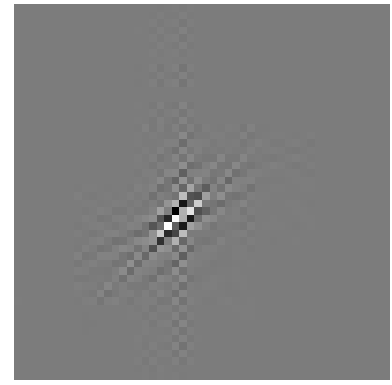

(a)

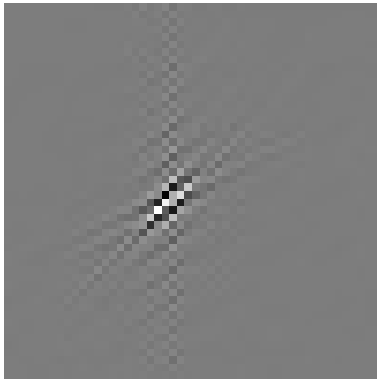

(b)

Figure 8: (a) Primal wavelet at the finest scale. (b) Associated dual wavelet.

As digital images lie in finite dimensional spaces, it is very unlikely that the obtained discrete wavelet system, drawn near to the Shannon wavelet basis, does not form a basis. The question whether it is a Riesz basis does not arise here. The point is rather to know whether the basis is close to an orthonormal basis or, in other words, whether the wavelet transform $W$ is close to an orthogonal transform. To this end, we compare the transform $W \circ W^{*}$ to the identity map. Figure 9 shows a detail of Barbara image (a) and its range by $W \circ W^{*}$ when (b) $\widehat{\psi}$ is as in $\mathbf{H 2}$ and (c) $\widehat{\psi}=|\widehat{\psi}|$. In the second case, the wavelet still generates an orthonormal basis for $W_{j, k}$, but the latter is no longer orthogonal to $W_{j, k+1}$. We can also surmise that the angle between $W_{j}$ and $W_{j+1}$ is smaller. When it is compared to (c), image (b) looks clearly very close to the original image (a). The PSNR is however quite low because of a low frequency errors.

The approximation of Barbara image with the $10^{4}$ largest directional wavelet coefficients is displayed in Figure 10. For a comparison, the result obtained with 9/7-separable wavelets [7] and wrapping curvelets [3] is also shown. Directional wavelets are more efficient than separable wavelets near edges and on textures although one can notice artifacts in smooth areas. The over-complete curvelet transform is poorly efficient at this rate of compression.

\section{Conclusion and outlook}

We gave sufficient conditions for a wavelet function to generate a rotation invariant Riesz sequence or a Riesz basis for $L^{2}\left(\mathbb{R}^{2}\right)$. This study leaves however several open questions. First, it does not let us to know whether the sequence considered in Section 3.1 is global for $L^{2}\left(\mathbb{R}^{2}\right)$. More generally, we did not give an example of wavelet with fast decay that satisfies all the conditions to generates a basis for $L^{2}\left(\mathbb{R}^{2}\right)$. 


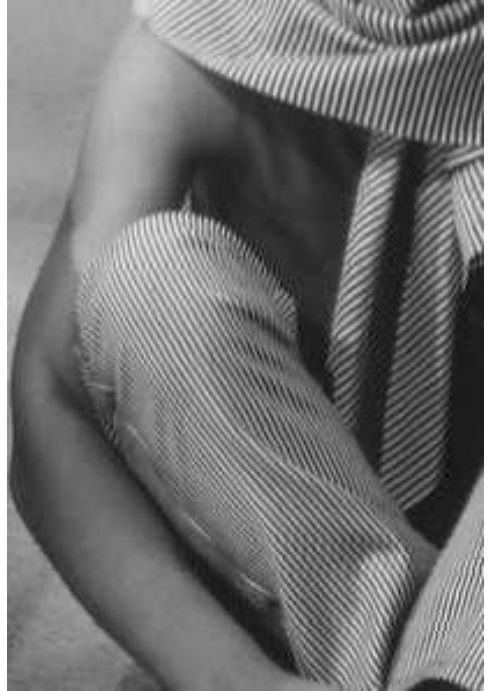

(a)

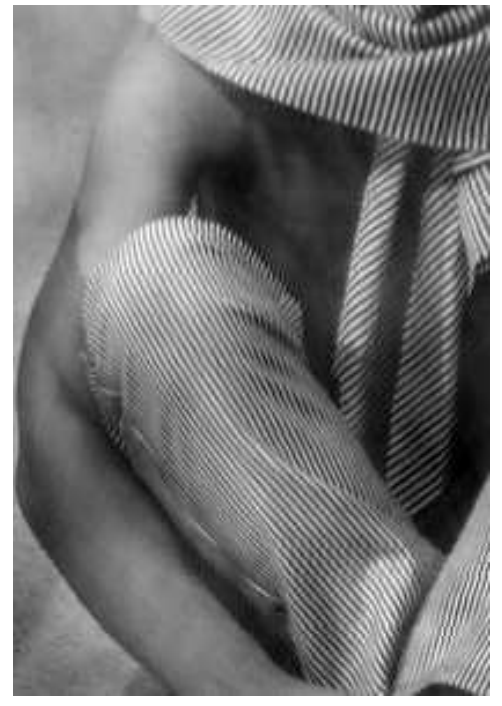

(b)

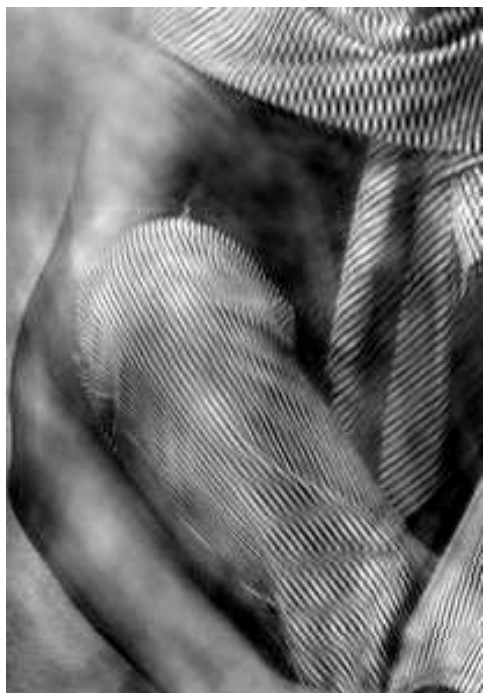

(c)

Figure 9: Barbara image and its range by $W \circ W^{*}$. (b) $\widehat{\psi}$ as in $\mathbf{H 2}(\operatorname{PSNR}=27.86 \mathrm{~dB})$. (c) $\widehat{\psi}=|\widehat{\psi}|$ $(\mathrm{PSNR}=21.21 \mathrm{~dB})$.

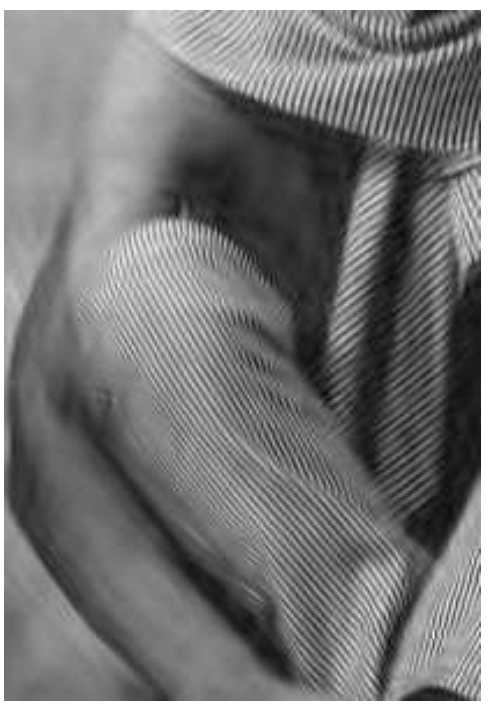

(a)

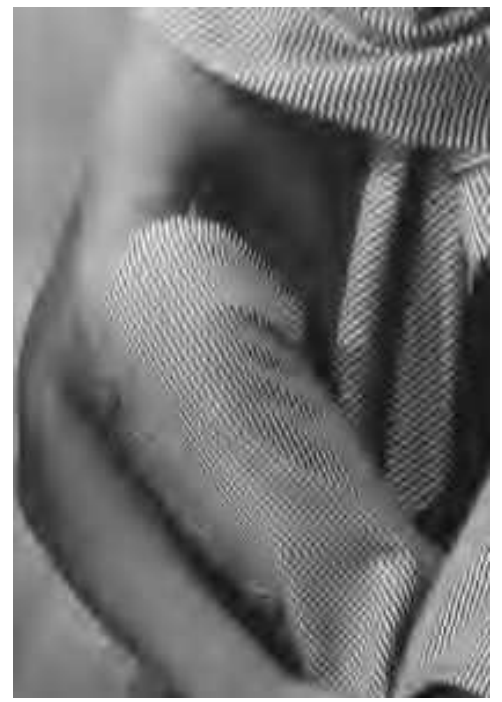

(b)

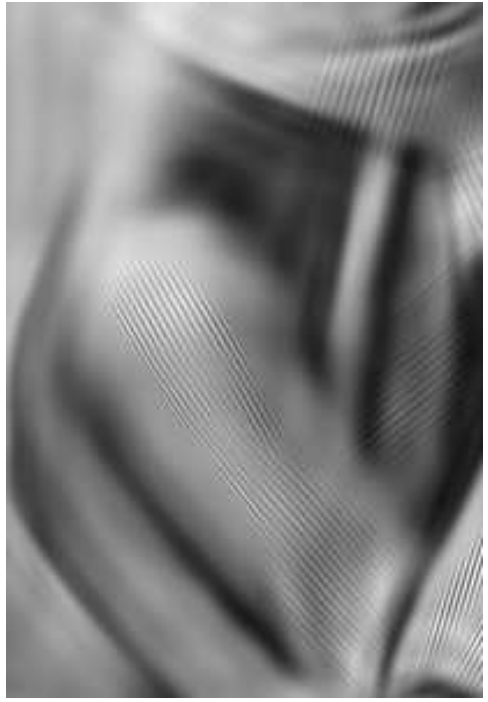

(c)

Figure 10: Approximation of Barbara image with the $10^{4}$ largest coefficients. (a) Proposed directional wavelets $(P S N R=28.31 \mathrm{~dB})$. (b) Separable wavelets $(P S N R=27.22 \mathrm{~dB})$. (c) Curvelets (PSNR=23.47 dB). 
Investigations could allow to find such an example. A more relevant way to obtain an analytic proof of the existence of such a basis would be however to find weaker assumptions in both theorems. It could be done by developing, for instance, a method that allows to estimate the upper bounds of Lemma 3 , and those of Lemma 4, as a whole.

The proposed implementation allows to keep the orthonormal basis for each space $W_{j}$ and numerical results let us surmise that angles between these spaces are fairly large. However, they impel us to consider several possible improvements. More adapted frequency tilings have already been mentioned and displayed in Figure 5. Stated theorems do not allow to apply a parabolic scaling law as in the curvelet transform, but since the two finest scales constitute $\frac{15}{16}$ of coefficients, we could obtain better approximations of images with sharp edges and textures by increasing the number of directions. This can be easily done, even with the proposed implementation for $2^{n} \times 2^{m}$-images. Finding a tiling that enables us to translate wavelets on sublattices of the original lattice $\mathbb{Z}^{2}$ would let us consider a proper processing of the finest scale and possibly compactly supported wavelets. The processing of the coarsest scale has also to be reconsidered. The design of dual wavelets, in the discrete as well as the continuous case, is a another challenging issue.

As a conclusion, we need to mention the very new manuscript by Yin and Daubechies [32]. To our knowledge, this is the only other article that proposes a method to circumvent the aliasing problem generated by filterbanks in directional wavelet bases. Although their approach - that relies on the nonuniform filterbank of $[26,11]$ - is very different to ours, several parallels can be drawn. For instance, they also consider biothogonal wavelets in order to design non-aliased primal wavelets and give conditions to properly chose the phase of their Fourier transforms.

\section{Acknowledgement}

The author thanks Joan Glaunès for a discussion leading to the proof of Lemma 5.

\section{References}

[1] J-P. Antoine and R. Murenzi, Two-dimensional directional wavelets and the scale-angle representation, Signal Processing 52: 259-281, 1996.

[2] J.D. Blanchard and I.A. Krishtal, Matricial filters and crystallographic composite dilation wavelets, Math. Comp. 81: 905-922, 2012.

[3] E. J. Candès, L. Demanet, D. L. Donoho and L. Ying, Fast Discrete Curvelet Transform, SIAM Multiscale Model. Simul. 5(3): 861-899, 2006.

[4] E. J. Candès and D. L. Donoho, Ridgelets - a key to higher dimensional intermittency ?, Phil. Trans. R. Soc. Lond. A., pp:2495-2509, 1999.

[5] E. J. Candès and D. L. Donoho, Curvelets - a surprizingly effective nonadaptive representation for objects with edges, Curve and Surface Fitting, Vanderbuilt Univ. Press, 1999.

[6] Consultative Committee for Space Data Systems, CCSDS 121.0-B-1: Lossless Data Compression, Blue Book, issue 1, May 1997. 
[7] A. Cohen, I. Daubechies and J. C. Feauveau, Biorthogonal bases of compactly supported wavelets, Comm. in Pure and Appl. Math., 45(5):485-560, 1992.

[8] A. Cohen and J. M. Schlenker, Compactly supported bidimensional wavelet bases with hexagonal symmetry, Constructive Approximation, 9:209-236, 1993.

[9] M. N. Do and M. Vetterli, Contourlets, Beyond Wavelets, Academic Press, New York, 2003.

[10] D. L. Donoho, Orthonormal ridgelets and linear singularities, SIAM Journal on Math. Analysis, 31(5):1062-1099, 2000.

[11] S. Durand, Orthonormal bases of non-separable wavelets with sharp directions, in proc. IEEE Int. Conf. on Image Proc., 2005.

[12] S. Durand, M-band filtering and non-redundant directional wavelets, Applied and Comput. Harmonic Analysis, 22(1):124-139, 2007.

[13] S. Durand, On the construction of discrete directional wavelets: HDWT, space-frequency localization and redundancy factor, SIAM Multiscale Modeling and Simulation 7(3):1325-1347, 2009.

[14] K. Guo, G. Kutyniok, D. Labate, Sparse multidimensional representations using anisotropic dilation and shear operators, Wavelets and Splines, G. Chen and M.J. Lai Eds, Nashboro Press: 189-201, 2006 .

[15] K. Guo, D. Labate, W. Q. Lim, G. Weiss and E. Wilson, Wavelets with composite dilations and their MRA properties, Applied and Comput. Harmonic Analysis, 20: 202-236, 2006.

[16] K. Guo, D. Labate, W. Q. Lim, G. Weiss and E. Wilson, The theory of wavelets with composite dilations, Harmonic Analysis and Applications, C. Heil ed., 231-249, Birkauser, 2006.

[17] A. Kiely and M. Klimesh, The ICER Progressive Wavelet Image Compressor, IPN Progress Report 42-155, 2003.

[18] I.A. Kristhal, B.D. Robinson, G.L. Weiss and E.N. Wilson, Some simple Haar-type wavelets in higher dimensions, J. Geom. Anal., 17(1): 87-96, 2007.

[19] ISO/IEC 15444-1:2000. Information technology JPEG 2000 image coding system. Part 1: Core coding system, 2000.

[20] N. G. Kingsbury, The dual-tree complex wavelet transform: A new technique for shift invariance and directional filters, in proc. IEEE DSP, 1998.

[21] J. MacArthur ans K.F. Taylor, Wavelets with Crystal Symmetry Shifts, J. Fourier Anal. Appl., 17: 1109-1118, 2011.

[22] U. Molter and A. Quintero, Crystallographic Multiwavelets in $L^{2}\left(\mathbb{R}^{d}\right)$ arXiv:1610.09019, 2016.

[23] E. Le Pennec and S. Mallat, Sparse geometrical image representation with bandelets, IEEE Trans. on Image Processing, 14(4): 423-439, 2005. 
[24] S. Mallat and G. Peyré, Orthogonal Bandlet Bases for Geometric Images Approximation, Comm. on Pure and Applied Math., 61(9): 1173-1212, 2008.

[25] R. Murenzi, Ondelettes multidimensionnelles et applications à l'analyse d'images, $\mathrm{PhD}$ Thesis, Univ. Cath. Louvain, Belgium, 1990.

[26] T. T. Nguyen and S. Oraintara, Multiresolution Direction Filter Banks: Theory, Design and Applications, IEEE Trans. Signal Proc., 3895-3905, 2005.

[27] I. W. Selesnick, The double-density dual-tree DWT, IEEE Trans. Signal Proc., 52(5): 1304-1314, 2004.

[28] E. P. Simoncelli and E. H. Adelson, Subband Image Coding with Hexagonal Quadrature Mirror Filters, Picture Coding Symposium, Cambridge, MA, 1990

[29] E. P. Simoncelli, W. T. Freeman, E. H. Adelson and D. J. Heeger, Shiftable Multiscale Transforms, IEEE Trans. Info. Theory, 38(2): 587-607, 1992

[30] R. Van Spaendonck, T. Blu, R. Baraniuk and M. Vetterli, Orthogonal Hilbert transform filter banks and wavelets, in proc. IEEE Int. Conf. on Acoustics, Speech and Signal Proc., 2003.

[31] R. Yin, Construction of Orthonormal Directional Wavelets based on quincunx dilation subsampling in proc. IEEE SampTA, 2015

[32] R. Yin and I. Daubechies Directional Wavelet Bases Constructions with Dyadic Quincunx Subsampling arXiv:1602.05469, 2016. 